\title{
Multifrequency study of HH 137 and HH 138: Discovering new knots and molecular outflows with Gemini ${ }^{\star}$ and APEX $\dagger$
}

\author{
Leticia V. Ferrero ${ }^{1,2} \neq$, Cristina E. Cappa ${ }^{2,3}$, Hugo P. Saldaño ${ }^{1}$ Mercedes Gómez $^{1,2}$, \\ Mónica Rubio ${ }^{4}$, and Guillermo Günthardt ${ }^{1}$ \\ ${ }^{1}$ Universidad Nacional de Córdoba, Observatorio Astronómico de Córdoba, Laprida 854, Córdoba X5000BGR, Argentina \\ ${ }^{2}$ Consejo Nacional de Investigaciones Científicas y Técnicas (CONICET), C1033AAJ, Argentina \\ ${ }^{3}$ Facultad de Ciencias Astronómicas y Geofísicas, Universidad Nacional de la Plata, Paseo del Bosque s/n, 1900 La Plata, Argentina \\ ${ }^{4}$ Departamento de Astronomía, Universidad de Chile, Casilla 36-D, Santiago, Chile
}

Accepted XXX. Received YYY; in original form ZZZ

\begin{abstract}
We present a multi-wavelength study of two HH objects (137 and 138) that may be associated. We use Gemini $\mathrm{H}_{2}(2.12 \mu \mathrm{m})$ and $\mathrm{K}(2.2 \mu \mathrm{m})$ images, as well as APEX molecular line observations and Spitzer image archives. Several $\mathrm{H}_{2}$ knots, linked to the optical chain of knots of $\mathrm{HH} 137$, are identified in the Gemini and Spitzer $4.5 \mu \mathrm{m}$ images. New shock excited regions related to the optical knots delineating $\mathrm{HH} 138$ are also reported. In addition, a bright $4.5 \mu \mathrm{m}$ 0.09 pc-long arc-shaped structure, roughly located mid-way between $\mathrm{HH} 137$ and $\mathrm{HH} 138$, is found to be associated with two Spitzer Class I/II objects, which are likely to be the exciting stars. These sources are almost coincident with a high-density molecular clump detected in ${ }^{12} \mathrm{CO}(3-2),{ }^{13} \mathrm{CO}(3-2), \mathrm{C}^{18} \mathrm{O}(3-2), \mathrm{HCO}^{+}(3-2)$ and $\mathrm{HCN}(3-2)$ molecular lines with an LTE mass of $36 \mathrm{M}_{\odot}$. The ${ }^{12} \mathrm{CO}(3-2)$ emission distribution over the observed region reveals molecular material underlying three molecular outflows. Two of them (outflows 1 and 2) are linked to all optical knots of $\mathrm{HH} 137$ and $\mathrm{HH} 138$ and to the $\mathrm{H}_{2}$ and $4.5 \mu$ m shock emission knots. In fact, the outflow 2 shows an elongated ${ }^{12} \mathrm{CO}$ blue lobe that coincides with all the $\mathrm{H}_{2}$ knots of $\mathrm{HH} 137$ which end at a terminal $\mathrm{H}_{2}$ bow shock. We propose a simple scenario that connects the outflows to the dust clumps detected in the region. A third possible outflow is located to the north-east projected towards a secondary weak and cold dust clump.
\end{abstract}

Key words: stars: jets - ISM: Herbig-Haro objects — ISM: jets and outflows - infrared: ISM — submillimeter: ISM — ISM: individual objects: HH 137 (MHO 1629), HH 138

\section{INTRODUCTION}

Stellar jets and molecular outflows appear during the initial stages of star-formation when the incipient star+disk system begins to eject winds along the rotation axis that interact with the surrounding cloud. The high kinetic energy of jets (comparable to the gravitational binding energy of the core where the stars are forming) has the potential to disperse the entire envelope of the new stars

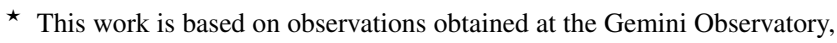
which is operated by the Association of Universities for Research in Astronomy, Inc., under a cooperative agreement with the NSF on behalf of the Gemini partnership: the National Science Foundation (United States), National Research Council (Canada), CONICYT (Chile), Ministerio de Ciencia, Tecnología e Innovación Productiva (Argentina), Ministério da Ciência, Tecnologia e Inovação (Brazil), and Korea Astronomy and Space Science Institute (Republic of Korea).

$\dagger$ APEX is a collaboration between the Max-Planck-Institute for Radio Astronomie, the European Southern Observatory, and the Onsala Space Observatory.

† Contact e-mail: 1vferrero@unc.edu.ar
(Tsinganos et al. 2009). Such an interaction can extend to a few parsecs away from the source. The jets can be observed over a wide range of wavelengths from the ultraviolet to the radio. In the optical they are evident as Herbig-Haro (HH) objects, while in the near infrared (NIR) they are observed as $\mathrm{H}_{2}(2.12 \mu \mathrm{m})$ knots (Reipurth \& Bally 2001; Bally 2016). In particular, the $\mathrm{H}_{2}$ line at $2.12 \mu \mathrm{m}$ is a well-known shock tracer, where the relatively fast jet hits and sweeps up the cold molecular cloud environment. This produces the molecular outflows which are detected at radio wavelengths, mainly in the ${ }^{12} \mathrm{CO}$ and $\mathrm{SiO}$ lines (Davis et al. 2010; Beuther et al. 2002; Arce et al. 2007; Maud et al. 2015).

The outflows are a ubiquitous phenomenon. They are present in objects within a wide range of masses from brown dwarfs (e.g., Riaz et al. 2017; Whelan et al. 2018) to massive protostars (e.g., Fedriani et al. 2018; McLeod et al. 2018). Such outflows broaden the molecular lines since they increase the motions within the cloud introducing non-Guassian wings into the ${ }^{12} \mathrm{CO}$ spectra. The outflows occur simultaneously with the infall motion, where the protostars are forming. The infall motion is revealed by means of 
double-peaked ${ }^{12} \mathrm{CO}$ (or $\mathrm{HCO}^{+}$) line profiles with the blue-shifted peak brighter than the red one (Evans 1999). The depression between both sides almost coincides with an optically thin line (such as $\mathrm{C}^{18} \mathrm{O}$ ), which frequently shows a single component peaking at the systemic velocity (e.g. Zhou \& Evans 1994; Cunningham et al. 2018).

Jets not only affect the nearby molecular cloud morphologically, but they also heat and compress the gas rapidly, triggering different molecular processes (such as molecular dissociation, sublimation of grain mantle ices, etc.) contributing to the chemical enrichment of the vicinity of young stars (Tsinganos et al. 2009). Thus, jets and outflows have significant consequences in the evolution of the environments of new stars. Although they have been widely studied in the literature, few of them have been observed at high-resolution in the infrared wavelengths and studied in a wide spectral range. Here, we present a new study of the $\mathrm{HH}$ objects named as HH 137 and HH 138 using infrared high-resolution observations obtained with the Gemini South telescope and radio observations of molecular emissions $\left(\mathrm{CO}\right.$ isotopologues, $\left.\mathrm{HCO}^{+}, \mathrm{HCN}\right)$ taken with the APEX telescope.

Both $\mathrm{HH}$ objects are located towards the dark nebula D291.4-0.2 ${ }^{1}$ (Hartley et al. 1986), in the Carina region. This highly dense dark cloud is $4^{\prime} \times 2^{\prime}$ or $\sim 2.5 \times 1.3 \mathrm{pc}$ (at a distance of $2.2 \mathrm{kpc}$, Moffat \& Vogt 1975; Steppe 1977) in size. It has been catalogued among the most opaque clouds studied by Sandqvist (1977) as well as by Hartley et al. (1986) and also detected in a high density molecular tracer such as $\mathrm{HCO}^{+} \mathrm{J}=1 \rightarrow 0$ by Barnes et al. (2011). In this latter work, this cloud is identified as BYF 129a. Figure 1 shows the $\mathrm{H} \alpha$ image towards D291.4-0.2 taken from the SuperCOSMOS Survey (Parker et al. 2005), where the dark cloud is observed as an opaque patch with no background stars detected (in $\mathrm{H} \alpha$ ) due to the high extinction $\left(A_{V}=13.5\right.$, Targon et al. 2011). The green dotted ellipses mark the positions of HH 137 and 138 . The fields observed with the Gemini South and the APEX telescope are indicated with yellow and white boxes, respectively. The image reveals that $\mathrm{HH} 137$ and 138 coincide with the dark patch.

These HH objects, identified in [S II], $\mathrm{H} \alpha$, and [N II] lines (Ogura 1993) show a chain of knots roughly in the East-West direction and they were named from A to $\mathrm{J}$ for $\mathrm{HH} 137$ and from A to D for HH 138. Ogura (1993) and Targon et al. (2011) have adopted a distance of $2.2 \mathrm{kpc}$ (Moffat \& Vogt 1975; Steppe 1977) for the two $\mathrm{HH}$ objects, and they estimated a size of $0.84 \mathrm{pc}$ for $\mathrm{HH} 137$ and 0.23 pc for HH 138. Nevertheless, Jackson et al. (2008), using the CS(2-1) high-density ( $n \gtrsim 10^{5} \mathrm{~cm}^{-3}$ ) molecular line tracer, derived a kinematic distance of $1.37 \mathrm{kpc}$. Other authors have obtained similar distances (see e.g, Kavars et al. 2005; Barnes et al. 2011; Planck Collaboration et al. 2016). Regarding the HH knots driving source, both Ogura (1993) and Targon et al. (2011) suggested that it should be located between HH 137-knot J and HH 138-knot A. However, it is not clear whether HH 138 and HH 137 are linked to the same unknown driving source.

The aim of this paper is to detect the near-infrared counterparts of these $\mathrm{HH}$ objects as well as to search for likely molecular outflows associated with these objects and derive their physical parameters. Our new infrared and molecular data allow us to analyze the molecular environment linked to the $\mathrm{HH}$ objects and to con-

1 D291.4-0.2 has also been catalogued as Globulet No. 127 (Sandqvist 1977) and No. 103 (Feitzinger \& Stuewe 1984), [DB2002b] G291.58+0.03 (Dutra \& Bica 2002), TGU H1803 (Dobashi et al. 2005) and DOBASHI 5797 (Dobashi 2011).

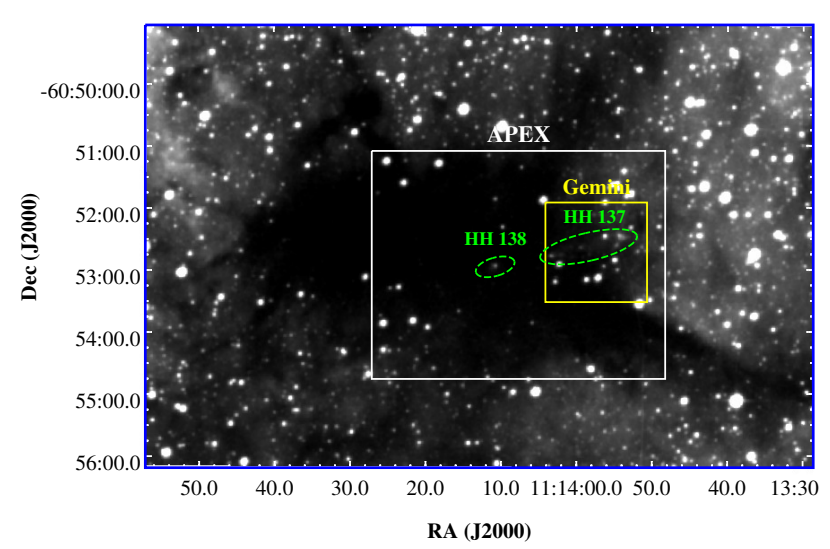

Figure 1. $\mathrm{H} \alpha$ image from the SuperCOSMOS Survey, where the areas observed with the Gemini (yellow square) and APEX (white square) telescopes are marked. The position of the knots associated with HH 137 and 138 are indicated with ellipses (dotted green lines).

tribute to a better understanding of the effect of outflows on the parent cloud.

The paper is outlined as follows. Our Gemini and APEX observations as well as Spitzer, WISE and Herschel archive data used in this work are described in $\S 2$. In $\S 3$, we show our Gemini results for HH 137. A complete analysis of HH 137 and HH 138 based on larger field mid- and far-IR archive images is presented in $\S 4$. The search for the potential driving source of these $\mathrm{HH}$ objects is carried out in $\S 5$. The analysis of the molecular line emissions and the identification of outflows that are likely linked to HH 137 and HH 138 are described in $\S 6$. In $\S 7$ the physical parameters of the outflows as well as a scenario for the outflows configuration are presented. Finally, in $\S 8$ we summarize the work and highlight the main results.

\section{DATA SETS}

\subsection{Gemini observations}

We used the NIR Gemini South Adaptive Optics Imager (GSAOI) and the Gemini Multi-conjugate Adaptive Optics System (GeMS), mounted at the 8-m Gemini South Telescope, in Cerro Pachón, Chile (McGregor et al. 2004; Carrasco et al. 2012). GeMS is an adaptive optic system that uses five sodium Laser Guide Stars (LSG), up to three Natural Guide Stars (NGS) and multiple deformable mirrors (DMs) that are optically conjugated with the main turbulence layers. This provides an adaptive optics (AO) corrected field that is larger than a single-conjugated adaptive optics (SCAO, Neichel et al. 2014a; Rigaut et al. 2014). GSAOI+GeMS provides diffraction limited images in the $0.9-2.4 \mu \mathrm{m}$ wavelength range over a $85^{\prime \prime} \times 85^{\prime \prime}$ field-of-view, with a imaging scale of 0 .' $^{\prime} 0197 \mathrm{pixel}^{-1}$. The GSAOI detector is composed by $2 \times 2$ Rockwell HAWAII- 2 RG $2048 \times 2048$ pixel array mosaic with four gaps between the arrays of $\sim 2.4 \mathrm{~mm}$, corresponding to $\sim 2.5^{\prime \prime}$ on the sky.

The images of HH137 were taken on 13th February 2014 (Program ID: GS-2014A-Q-29) in the $\mathrm{H}_{2}$ (1-0 $\left.\mathrm{S}(1), \lambda_{\mathrm{c}}=2.122 \mu \mathrm{m}, \Delta \lambda=0.032 \mu \mathrm{m}\right)$ and $\mathrm{K}\left(\lambda_{\mathrm{c}}=2.200 \mu \mathrm{m}\right.$, $\Delta \lambda=0.340 \mu \mathrm{m})$ filters. We used a $3 \times 3$ dither pattern with steps of $8^{\prime \prime}$ to remove the gaps between the detectors. We obtained a total of 9 science fields with individual exposure times of $100 \mathrm{sec}$ each in the $\mathrm{H}_{2}$ filter, and 9 science fields of $40 \mathrm{sec}$ each in the K-filter. Since 
it was possible to use three Natural Guide Stars (NGSs) with a good asterism geometry (Neichel et al. 2014a,b), the multi-conjugate adaptive optics (MCAO) performance was highly uniform over the field. The final images have a resolution of $0.09 \mathrm{arcsec} \mathrm{pixel}^{-1}$ which is in agreement with the values reported by Neichel et al. (2014a).

The images were processed and combined using THELI ${ }^{2}$ (Schirmer 2013; Erben et al. 2005). The reduction process was similar to that described in Schirmer (2013) and Schirmer et al. (2015). The science images were used to make a master sky image that, in turn, was subtracted. To obtain the astrometry, the 2MASS catalog was used. We estimated an average precision of 0.17 arcsec in our coordinates with respect to the 2MASS catalog.

\subsection{APEX observations}

We obtained ${ }^{12} \mathrm{CO}(3-2)$ (at $\left.345.796 \mathrm{GHz}\right),{ }^{13} \mathrm{CO}(3-2)$ (at $330.588 \mathrm{GHz}$ ), $\mathrm{C}^{18} \mathrm{O}(3-2)$ (at $\left.329.330 \mathrm{GHz}\right), \mathrm{HCO}^{+}(3-2)$ (at 267.557 GHz) and $\mathrm{HCN}(3-2)$ (at $265.886 \mathrm{GHz}$ ) molecular data using the 12-m Atacama Pathfinder EXperiment (APEX) telescope, located in the Llano de Chajnantor, Chile (Güsten et al. 2006). The observations were performed on 24th March 2016 and 17th to 20th June 2016 (Project 097.F-9707A-2016, PI: M. Rubio), using the On-The-Fly technique with a space between dumps in the scanning direction of $9^{\prime \prime}$. The off source position free of $\mathrm{CO}$ emission was RA.,Dec. $(\mathrm{J} 2000)=(11: 00: 50.8,-61: 00: 19.4)$. The observed region was centered between $\mathrm{HH} 137$ and $\mathrm{HH} 138$ in the coordinate RA.,Dec. $(\mathrm{J} 2000)=\left(11^{h} 14^{m} 07.38^{s},-60^{\circ} 52^{\prime} 53.34^{\prime \prime}\right)$ covering an area of $5^{\prime} \times 4^{\prime}$ for ${ }^{12} \mathrm{CO},{ }^{13} \mathrm{CO}$ and $\mathrm{C}^{18} \mathrm{O}$, and $3^{\prime} \times 3^{\prime}$ for $\mathrm{HCO}^{+}$ and $\mathrm{HCN}$.

The APEX-2 (SHeFI, Vassilev et al. 2008) receiver was used to observe the CO lines with a half-power beam width (HPBW) of $\sim 20^{\prime \prime}$, while the APEX-1 (SHeFI) receiver was employed to observe the $\mathrm{HCO}^{+}$and $\mathrm{HCN}$ line molecules with an HPBW of $\sim 24^{\prime \prime}$.

Raw data have a velocity resolution of $\sim 0.11 \mathrm{~km} \mathrm{~s}^{-1}$ at $345 \mathrm{GHz}$. The velocity resolution was decreased to $0.3 \mathrm{~km} \mathrm{~s}^{-1}$ to improve the signal to noise. Pointing and calibration were performed during the observations using L02 Pup, IRAS 07454-7112, IRAS 15194-5115, IRC +10216 and Carina sources. The calibration of the intensity has an uncertainty of $10 \%$.

The spectra were reduced using the Continuum and Line Analysis Single-dish Software (CLASS) of the Grenoble Image and Line Data Analysis Software (GILDAS ${ }^{3}$ ) according to the standard procedure of the CLASS software. The rms noise of the profiles after baseline subtraction and calibration is $0.2 \mathrm{~K}$. The observed line intensities are expressed as main-beam brightness temperatures $T_{m b}$ dividing the antenna temperature $T_{A}$ by the main-beam efficiency $\eta_{m b}$, equal to 0.72 for APEX-1 and APEX-2 (Vassilev et al. 2008). The Astronomical Image Processing System (AIPS) package as well as the CLASS software were used to perform the analysis.

2 THELI is a tool for the automated reduction of astronomical images in optical, near- and mid-infrared, available on the website: https://www . astro.uni-bonn. de/theli/.

3 GILDAS is available at: http://www.iram.fr/IRAMFR/ GILDAS /.

\subsection{Complementary data}

Images in the four IRAC bands centered at 3.6, 4.5, 5.8, and $8.0 \mu \mathrm{m}$ were obtained from the Galactic Legacy Infrared Mid-Plane Survey Extraordinaire (GLIMPSE; Benjamin et al. 2003). Near- and midIR images at 3.4, 4.6, 12 and $22 \mu \mathrm{m}$ were taken from the ALLWISE Data Release (Wright et al. 2010). Far-IR data from the Herschel Infrared GALactic Plane Survey (Hi-GAL, Molinari et al. 2010) at 70 and $160 \mu \mathrm{m}$ (PACS, Poglitsch et al. 2010), and at $250 \mu \mathrm{m}$ (SPIRE, Griffin et al. 2010) were also used. These data have angular resolutions of $7^{\prime \prime}, 11^{\prime \prime}$ and $18^{\prime \prime}$, respectively. The APEX Telescope LArge Survey of the GALaxy (ATLASGAL ${ }^{4}$, Schuller et al. $2009)$ at $870 \mu \mathrm{m}(345 \mathrm{GHz})$ was also employed (beam size 19.' 2). This survey has an rms noise in the range of 0.05-0.07 Jy beam ${ }^{-1}$ and a calibration uncertainty of $15 \%$. In addition, optical images from SuperCOSMOS digitised data of the AAO/UKST H $\alpha$ survey (SHS ${ }^{5}$, Parker et al. 2005) of the Southern Galactic Plane were used to construct Figure 1.

\section{GEMINI RESULTS}

Figure 2 shows a composite image of $\mathrm{HH} 137$ in $\mathrm{K}$ (magenta) and $\mathrm{H}_{2}$ (green) in a $98^{\prime \prime} \times 96^{\prime \prime}$ field. The chain of knots linked to HH 137 is seen in shock excited $\mathrm{H}_{2}$ emission. Enlarged sectors of Figure 2 are displayed in panels $a$ ) to $d$ ). The pink crosses mark the position of optical knots listed by Ogura (1993) with the nomenclature used therein. The new $\mathrm{H}_{2}$ knots are designated as MHO 1629 in the on-line Catalogue of Molecular Hydrogen Emission-Line Objects $^{6}$ (MHOs, Davis et al. 2010). We assigned capital letters from $\mathrm{K}$ to $\mathrm{O}$ to the new structures, with no apparent optical counterparts. They are highlighted by yellow circles or an ellipse in Figure 2 (see also Ferrero et al. 2015). These new $\mathrm{H}_{2}$ structures are more clearly seen in panels $a$ ), $b$ ) and $d$ ). Optical and NIR knots do not strictly coincide as it has been found in other jets (e.g., Davis et al. 1994; Reipurth et al. 2000). Assuming that the exciting source is located between knot HH 137-J and HH 138-A (Ogura 1993), the opening angle $(\theta)$ of $\mathrm{HH} 137$ is $\sim 13.2^{\circ}$ and the collimation factor $^{7} R_{\text {coll }} \sim 5$. We measure a PA of $284^{\circ}$ in agreement with Ogura $\left(1993, \mathrm{PA} \sim 283^{\circ}\right)$.

Table 1 provides a cross identification between the knots of Ogura (1993) and the $\mathrm{H}_{2}$ emissions in Figure 2. Considering that the width of the $\mathrm{H}_{2}$ filter is about 10 times smaller than the width of the K-band filter, we calibrated in flux our $\mathrm{H}_{2}$ (1-0) $\mathrm{S}(1)$ image using the 2MASS $\mathrm{K}_{s}$ band magnitudes of 9 field stars. The fluxes were measured taking into account a $3 \sigma$ threshold between the background $\mathrm{rms}$ and $\mathrm{H}_{2}$ emission. The knots coordinates and final $\mathrm{H}_{2}$ fluxes are listed in fourth, fifth and sixth columns. The parameter $r$ (in the seventh column) is the size (radius in arcsec) of the circular area used for the aperture photometry of each knot. To estimate the errors, the uncertainties resulting from the conversion factor derived from the 2 MASS $\mathrm{K}_{s}$ magnitudes and the sky background variations in the $\mathrm{H}_{2}$ filter were taken into account.

4 ATLASGAL survey is available at: http://atlasgal. mpifr-bonn.mpg.de/cgi-bin/ATLASGAL_DATABASE.cgi.

5 SHS is available at: http://www-wfau.roe.ac.uk/sss/ halpha/.

6 http://astro.kent.ac.uk/ df/MHCat/

7 The collimation factor $R_{\text {coll }}$ is defined as the ratio between the semi-major axis $R_{\max }$ and the semi-minor axis $R_{\min }$ of the outflow: $R_{\text {coll }}=R_{\max } / R_{\min }$ (see e.g., Lada 1985). 

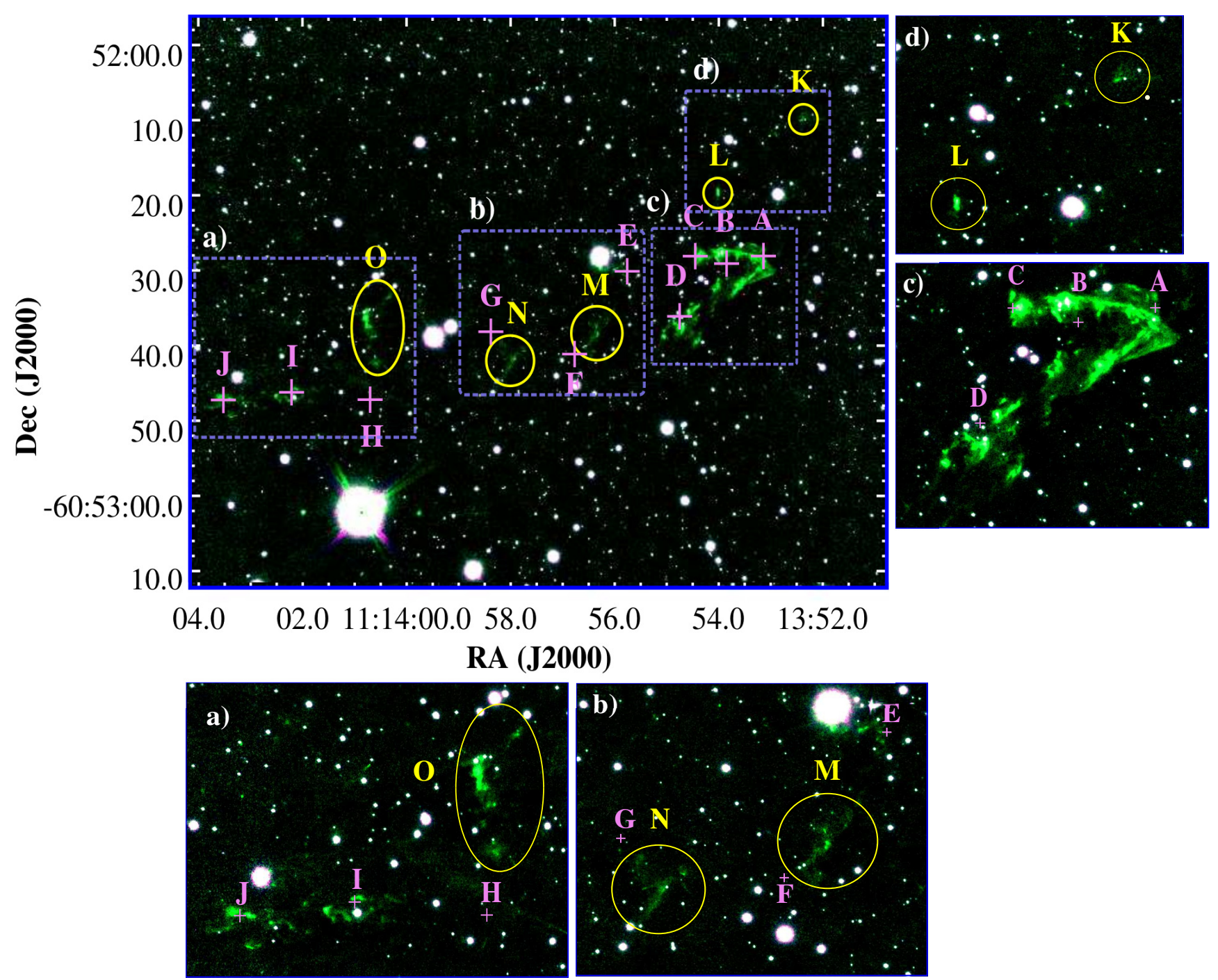

Figure 2. Composite image of $\mathrm{HH} 137$ showing $\mathrm{K}$ (magenta) and $\mathrm{H}_{2}$ (green) filters taken with GSAOI+GeMS. The pink crosses mark the positions of the optical knots detected by Ogura (1993). The yellow circles and the ellipse indicate five new $\mathrm{H}_{2}$ emission structures designated with the capital letters from $\mathrm{K}$ to $\mathrm{O}$, belonging to MHO 1629. Enlarged sectors of the GSAOI+GeMS image, labeled as panels $a$ ) to $d$ ), show more clearly the new $\mathrm{H}_{2}$ knots, detected in this work as well as the $\mathrm{H}_{2}$ counterparts of the $\mathrm{HH} 137$ optical knots. In particular panel c) displays the terminal bow-shock shape, coinciding with HH 137-A, B, $\mathrm{C}$ and $\mathrm{D}$.

The high angular resolution obtained with GSAOI+GeMS/Gemini (see Figure 2) reveals the complex internal structure of the knots. The knots HH 137-A to C display the typical terminal "bow-shock" shape more clearly than Ogura (1993, see panel $c$ )). The panel $a$ ) shows the HH 137-H to J knots in more detail, whereas panel $b$ ) displays HH 137 knots E, F and $\mathrm{G}$. We note that knots $\mathrm{G}$ and $\mathrm{H}$ are barely detectable in the Ogura's images. It is remarkable that the spatial separation between knot $\mathrm{H}$ listed by Ogura (1993) and the emission in $\mathrm{H}_{2}$, identified as $\mathrm{O}$, is of 10 .' 6 . For knot $\mathrm{G}$ and the nearest $\mathrm{H}_{2}$ emission $(\mathrm{N})$ a similar separation (of about $5^{\prime \prime}$ ) is observed. These separations are not easily reconcilable with any velocity expected for these type of objects (see e.g., Bachiller \& Tafalla 1999; Noriega-Crespo \& Garnavich 2001; Reipurth \& Bally 2001; Graham et al. 2003; McGroarty et al. 2007; Bally 2016).

\section{IR DATA ANALYSIS}

The upper panel of Figure 3 displays a composite image of the Spitzer emission at 3.6 (in blue), 4.5 (in green) and $8.0 \mu \mathrm{m}$ (in red). Optical knots associated with HH 137 and 138 from Ogura (1993) are marked with crosses (magenta and white crosses, respectively), while the yellow circles indicate the new $\mathrm{H}_{2}$ knots detected (see Figure 2). Several bright $4.5 \mu \mathrm{m}$ emissions are evident, most of them coinciding with Ogura's knots and/or the $\mathrm{H}_{2}$ knots here reported associated with HH 137. The optical knots HH 137-A to D belonging to the $\mathrm{H}_{2}$ bow-shock region are the most prominent at $4.5 \mu \mathrm{m}$. An arclike structure located between HH 137 and HH 138 is clearly seen at $4.5 \mu \mathrm{m}$. This structure has not been previously reported. Thus, we note it for the first time and name it as the $4.5 \mu \mathrm{m}$ arc. The projected size of the arc is of about $12^{\prime \prime}(0.09 \mathrm{pc}$ 
Table 1. HH 137 knots detected by Ogura (1993) and in $\mathrm{H}_{2}$ knots in our Gemini image.

\begin{tabular}{|c|c|c|c|c|c|c|c|}
\hline \multicolumn{3}{|c|}{ Ogura (1993) } & \multicolumn{5}{|c|}{ This work } \\
\hline HH 137 & $\begin{array}{l}\text { R.A.(J2000.0) } \\
\left({ }^{h}:{ }^{m}: s^{s}\right)\end{array}$ & $\begin{array}{l}\text { Dec.(J2000.0) } \\
\left({ }^{\circ}:{ }^{\prime}:{ }^{\prime \prime}\right)\end{array}$ & MHO 1629 & $\begin{array}{l}\text { R.A.(J2000.0) } \\
\left({ }^{h}: m^{\prime}: s\right)\end{array}$ & $\begin{array}{l}\text { Dec.(J2000.0) } \\
\left({ }^{\circ}::^{\prime \prime}\right)\end{array}$ & $\begin{array}{c}F_{H_{2}} \\
\left(10^{-5} \mathrm{Jy}\right)\end{array}$ & $\begin{array}{c}\mathrm{r} \\
\left({ }^{\prime \prime}\right)\end{array}$ \\
\hline A & $11: 13: 53.14$ & $-60: 52: 28.08$ & - & - & - & - & - \\
\hline - & - & - & A-1 & $11: 13: 53.42$ & $-60: 52: 30.25$ & $28.8 \pm 5.2$ & 4.09 \\
\hline - & - & - & A-2 & $11: 13: 54.03$ & $-60: 52: 32.21$ & $3.0 \pm 0.9$ & 1.33 \\
\hline B & $11: 13: 53.85$ & $-60: 52: 29.09$ & B & $11: 13: 53.98$ & $-60: 52: 27.83$ & $10.6 \pm 1.0$ & 1.49 \\
\hline $\mathrm{C}$ & $11: 13: 54.45$ & $-60: 52: 28.10$ & $\mathrm{C}$ & 11:13:54.39 & $-60: 52: 28.11$ & $6.9 \pm 0.4$ & 1.51 \\
\hline D & 11:13:54.75 & $-60: 52: 36.11$ & D & 11:13:54.70 & $-60: 52: 37.42$ & $14.9 \pm 0.3$ & 3.74 \\
\hline $\mathrm{E}$ & 11:13:55.76 & $-60: 52: 30.12$ & $\mathrm{E}^{a}$ & 11:13:55.90 & $-60: 52: 30.26$ & - & - \\
\hline $\mathrm{F}$ & 11:13:56.77 & $-60: 52: 41.14$ & - & - & - & - & - \\
\hline G & 11:13:58.38 & $-60: 52: 38.16$ & - & - & - & - & - \\
\hline $\mathrm{H}$ & 11:14:00.70 & $-60: 52: 47.20$ & - & - & - & - & - \\
\hline I & 11:14:02.21 & $-60: 52: 46.22$ & I & 11:14:02.22 & $-60: 52: 46.82$ & $4.1 \pm 0.3$ & 2.84 \\
\hline $\mathrm{J}$ & 11:14:03.52 & $-60: 52: 47.24$ & $\mathrm{~J}$ & 11:14:03.37 & $-60: 52: 49.34$ & $4.1 \pm 1.0$ & 4.17 \\
\hline - & - & - & $\mathrm{K}^{b}$ & $11: 13: 52.43$ & $-60: 52: 10.12$ & $1.0 \pm 0.1$ & 1.20 \\
\hline - & - & - & $\mathrm{L}^{b}$ & $11: 13: 54.03$ & $-60: 52: 19.45$ & $1.8 \pm 0.1$ & 1.02 \\
\hline - & - & - & M & 11:13:56.51 & $-60: 52: 39.80$ & $2.1 \pm 0.5$ & 3.11 \\
\hline - & - & - & $\mathrm{N}$ & 11:13:58.06 & $-60: 52: 42.15$ & $2.3 \pm 0.7$ & 3.02 \\
\hline- & - & - & $\mathrm{O}$ & 11:14:00.73 & $-60: 52: 37.03$ & $6.7 \pm 0.3$ & 2.61 \\
\hline
\end{tabular}

Notes: ${ }^{a}$ Knot $\mathrm{E}$ was not measured as the $\mathrm{H}_{2}$ emission is contaminated by a close bright star.

${ }^{b}$ Knots $\mathrm{K}$ and L correspond to knots X2 and X1 in Ferrero et al. (2015).

Table 2. HH 138 knots detected by Ogura (1993) and new EGOs at $4.5 \mu \mathrm{m}$.

\begin{tabular}{|c|c|c|c|c|c|c|}
\hline \multirow[b]{2}{*}{ Knot } & \multicolumn{2}{|c|}{ Ogura (1993) } & \multicolumn{4}{|c|}{ This work } \\
\hline & $\begin{array}{l}\text { R.A.(J2000.0) } \\
\left(h:^{m}: s\right)\end{array}$ & $\begin{array}{l}\text { Dec.(J2000.0) } \\
\left({ }^{\circ}::^{\prime}:{ }^{\prime \prime}\right)\end{array}$ & $\begin{array}{l}\text { R.A.(J2000.0) } \\
\left({ }^{h}: m: s\right)\end{array}$ & $\begin{array}{c}\text { Dec.(J2000.0) } \\
\left({ }^{\circ}::^{\prime}:{ }^{\prime \prime}\right)\end{array}$ & $\begin{array}{l}F_{[4.5]} \\
(\mathrm{mJy})\end{array}$ & $\begin{array}{c}\text { Area } \\
\left(\operatorname{arcsec}^{2}\right)\end{array}$ \\
\hline HH 138-A & 11:14:10.99 & $-60: 52: 56.35$ & $11: 14: 11.00$ & $-60: 52: 56.76$ & $479.7 \pm 20.5$ & 163.63 \\
\hline B & $11: 14: 11.29$ & $-60: 53: 00.35$ & $11: 14: 11.25$ & $-60: 53: 00.41$ & $54.6 \pm 4.1$ & 33.55 \\
\hline $\mathrm{C}$ & $11: 14: 13.00$ & $-60: 53: 03.38$ & $11: 14: 12.92$ & $-60: 53: 03.67$ & $274.9 \pm 1.6$ & 72.62 \\
\hline $\mathrm{D}$ & $11: 14: 13.61$ & $-60: 53: 02.39$ & $11: 14: 13.57$ & $-60: 53: 02.65$ & $369.2 \pm 1.2$ & 91.18 \\
\hline $4.5-\operatorname{arc}$ & - & - & 11:14:08.42 & $-60: 52: 55.36$ & $277.8 \pm 82.6$ & 296.73 \\
\hline HH 137-A-B-C & - & - & $11: 13: 53.70$ & $-60: 52: 29.47$ & $374.4 \pm 19.0$ & 452.74 \\
\hline
\end{tabular}

at $1.5 \mathrm{kpc}^{8}$, see Section 6.1 ), with the apex pointing roughly to the west.

Bright extended emissions in $4.5 \mu \mathrm{m}$ are usually classified as EGOs (Extended Green Objects, Cyganowski et al. 2008) or "Green Fuzzies" (Chambers et al. 2009). Since the $4.5 \mu \mathrm{m}$ band contains several rotovibrational $\mathrm{H}_{2}$ lines and $\mathrm{CO}$ band heads (Reach et al. 2006; Smith \& Rosen 2005; Watson et al. 2010), it is generally used as shock diagnostic. The fact that several $4.5 \mu \mathrm{m}$ emissions in Figure 3 spatially coincide with the $\mathrm{H}_{2}$ emissions in HH 137 (MHO 1629), and particularly with the HH 137 terminal bow-shock region, indicates collisional excited objects. Table 2 lists the HH 138 knots identified by Ogura (1993) and the new EGOs shown in Figure 3, upper panel. Consequently, even when our $\mathrm{H}_{2}$ image does not cover the $\mathrm{HH} 138$ region, the coincidence between 2.12 and $4.5 \mu \mathrm{m}$ emissions suggests that the $4.5 \mu \mathrm{m}$ emission is associated with jet emission (e.g., Smith et al. 2006; Davis et al. 2007; Cyganowski et al. 2008).

\footnotetext{
${ }^{8}$ Hereafter we will adopt a distance of $1.5 \mathrm{kpc}$ for $\mathrm{HH} 137$ and 138, as discussed in Section 6.1.
}

The emission at $8 \mu \mathrm{m}$ traces polycyclic aromatic hydrocarbons (PAHs) and photodissociation regions (PDRs, Watson et al. 2008). Emission at $8 \mu \mathrm{m}$ is identified towards the borders of the image and in coincidence with part of the terminal shock and some point-like sources close to the $4.5 \mu \mathrm{m}$ and optical knots of HH 138. The lack of extended $8 \mu \mathrm{m}$ emission close to the $\mathrm{HH}$ objects indicates that the region has a large visual absorption and shares some characteristics with infrared dark clouds (IRDC, Sanhueza et al. 2010). In fact, the GLIMPSE [3.6] and [4.5] $\mu \mathrm{m}$ mosaic image of the region clearly delineates a dark patch, coinciding with the optical extinction. Moreover, Simon et al. (2006a) had previously reported the detection of an IRDC named MSX-DC G291.40-0.19 based on $8.3 \mu \mathrm{m}$ mid-infrared image acquired with the Midcourse Space Experiment satellite.

The composite image in the bottom panel of Figure 3 displays Spitzer $4.5 \mu \mathrm{m}$ (in green), Herschel 70 and $250 \mu \mathrm{m}$ (in red and blue, respectively), and ATLASGAL $870 \mu \mathrm{m}$ (in contours) data. The dashed white ellipses mark the positions of HH 137 and HH 138. A bright isolated source is clearly detected at $70 \mu \mathrm{m}$, which coincides with the two point-like sources close to the $4.5 \mu \mathrm{m}$ arc (see the upper panel). The emission distribution from ATLASGAL reveals a 

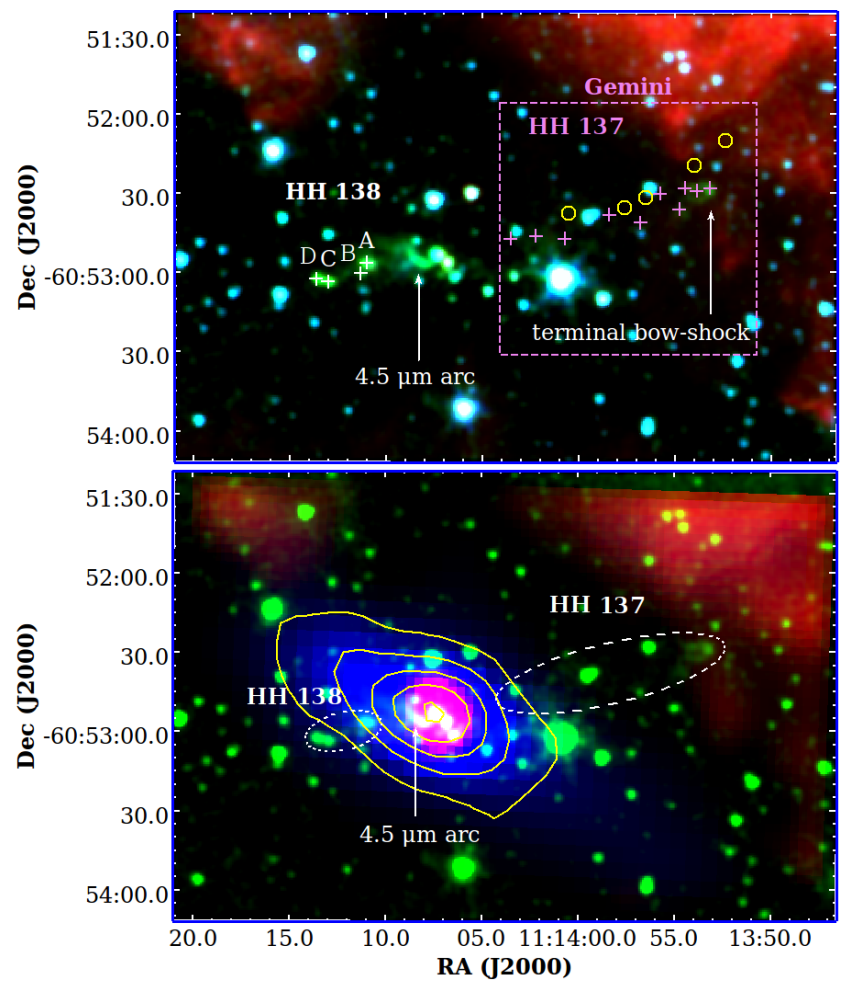

Figure 3. Upper panel: Composite image of the Spitzer emission at 3.6 (in blue), 4.5 (in green) and $8.0 \mu \mathrm{m}$ (in red). The crosses mark the positions of Ogura's knots and the circles the new $\mathrm{H}_{2}$ knots found in this work (MHO 1629). The location of the arc-like structure detected in $4.5 \mu \mathrm{m}$ and the location of $\mathrm{H}_{2}$ terminal bow shock are indicated as well as the area covered with Gemini (dashed magenta line, see Figure 2). Lower panel: Spitzer $4.5 \mu \mathrm{m}$, (in green), Herschel 70 and $250 \mu \mathrm{m}$ (in red and blue, respectively), and ATLASGAL $870 \mu \mathrm{m}$ (in yellow contours). Contours correspond to 40, $50,60,70$ and $80 \mathrm{Jy} \mathrm{beam}^{-1}$. The ellipses (dashed white line) indicate the location of HH 137 (on the right) and HH 138 (on the left).

cold dust clump (G291.367-00.214, Csengeri et al. 2014) centered on the arc at $4.5 \mu \mathrm{m}$ and a group of Spitzer point-like sources. The dust clump is $100^{\prime \prime} \times 46^{\prime \prime}$ or about $0.73 \mathrm{pc} \times 0.33 \mathrm{pc}$ at $1.5 \mathrm{kpc}$ ). This clump is classified as a protostellar region since it contains several IR sources detected at $4.5 \mu \mathrm{m}$ (Chambers et al. 2009).

Using ATLASGAL $870 \mu \mathrm{m}$ data, the dust mass of the clump can be estimated applying the expression by Hildebrand (1983):

$\mathrm{M}_{\text {dust }}=\frac{S_{870} d^{2}}{\kappa_{870} B_{870}\left(T_{\text {dust }}\right)}$,

where $S_{870}$ is the flux density at $870 \mu \mathrm{m}, d=1.5 \pm 0.5 \mathrm{kpc}$, $\kappa_{870}=2.06 \mathrm{~cm}^{2} / \mathrm{g}$ is the dust opacity per unit mass (Ossenkopf \& Henning 1994) and $B_{870}\left(T_{\text {dust }}\right)$ is the Planck function at a temperature $T_{\text {dust }}$. The flux density, $S_{870}$, of the clump was obtained integrating the observed emitting area within an aperture of $45^{\prime \prime}$ (radius), resulting in a flux of $6.0 \pm 0.5 \mathrm{Jy}$. The subtracted background emission was estimated in an annulus with inner and outer radii of $90^{\prime \prime}$ and $140^{\prime \prime}$, respectively.

To estimate $T_{\text {dust }}$ of the clump, we used temperature as well as column density maps from Marsh et al. (2017). These maps ${ }^{9}$ were produced employing the point process mapping (PPMAP) method

\footnotetext{
9 http://www.astro.cardiff.ac.uk/research/ ViaLactea/
}
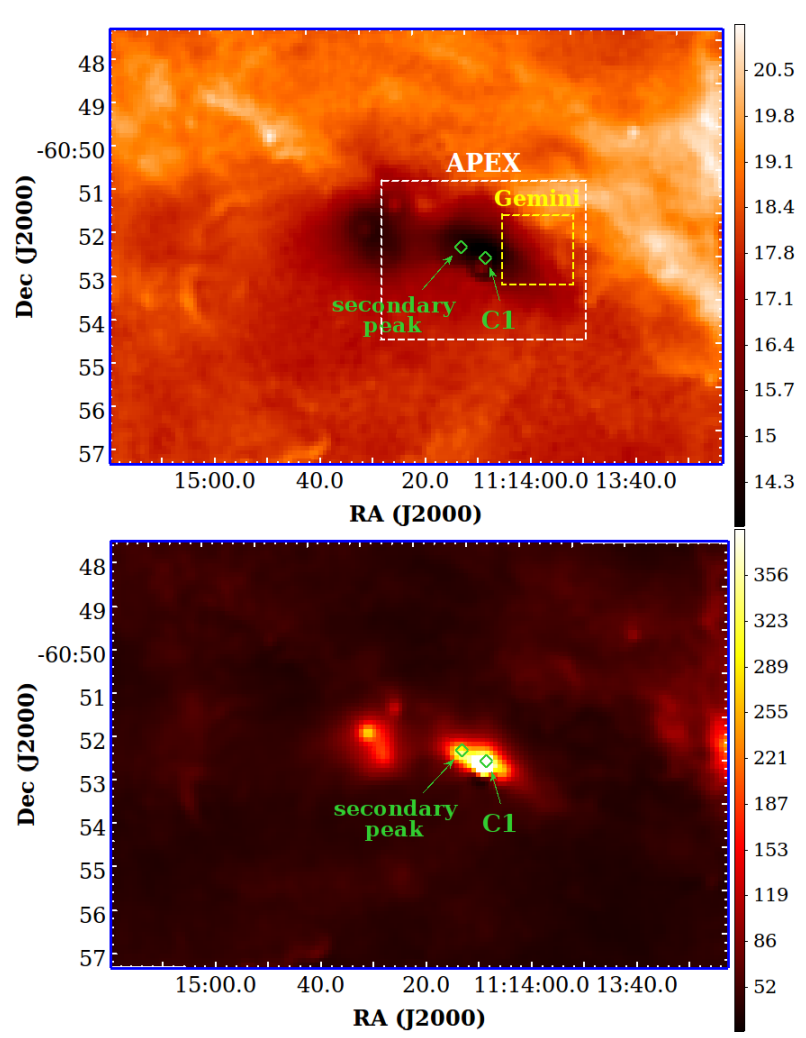

Figure 4. Dust temperature (upper panel) and column density (lower panel) maps in an area of $14^{\prime} \times 10^{\prime}$ centered at RA.,Dec. $(J 2000)=(11: 14: 19.704$, -60:52:36.78), obtained from Marsh et al. (2017). Two structures are marked: $\mathrm{C} 1$, discussed in this section (Section 4), and a secondary peak within the area of $\mathrm{C} 1$, introduced in Section 5. In the upper panel, the dashed yellow line box indicates the field observed with Gemini and the dashed white line box the field covered with APEX. Color scales are in units of K and $10^{20} \mathrm{~cm}^{-2}$ for the temperature and column density maps, respectively.

based on a Bayesian procedure applied to Herschel continuum data in the wavelength range $70-500 \mu \mathrm{m}$ (Marsh et al. 2015). This method provides resolution-enhanced $\left(\sim 12^{\prime \prime}\right)$ images of dust temperature and column density. Figure 4 shows both the temperature and column density $\left(\mathrm{N}\left(\mathrm{H}_{2}\right)\right)$ maps, in an area of $14^{\prime} \times 10^{\prime}$ centered at RA.,Dec. $(\mathrm{J} 2000)=(11: 14: 19.704,-60: 52: 36.78)$. In both panels of Figure 4 the dust clump $\mathrm{C} 1$ is marked. A secondary weaker dust peak is detected within the area of $\mathrm{C} 1$.

The temperature within the area of the dust clump varies from 13.5 to $16.2 \mathrm{~K}$. Adopting an average temperature of $14.8 \pm 0.6 \mathrm{~K}$, the dust mass turns out to be $M_{\text {dust }}=1.2 \pm 0.5 \mathrm{M}_{\odot}$. For a standard gas-to-dust ratio, $R$ of 100 (e.g., Elia et al. 2013, 2017; König et al. 2017), the hydrogen gas mass amounts to $M_{H_{2}}=120 \pm 50 \mathrm{M}_{\odot}$. It is worth mentioning that the quoted uncertainty in the hydrogen mass neglects any known factors in $R$ and/or $\kappa_{870}$. We also used the dust column density map from Marsh et al. (2017), shown in Figure 4 lower panel, to estimate $M_{H_{2}}$. To do this, we integrated over the area delimited by the contour of $8.7 \times 10^{21} \mathrm{~cm}^{-2}$ at $3 \sigma$. This contour comprises an area similar to that used to calculate the flux at $870 \mu \mathrm{m}$. The hydrogen gas mass turns out to be $M_{H_{2}}=175 \pm 21 \mathrm{M}_{\odot}$.

The discrepancy between the two values of mass is larger than the quoted uncertainties. Nonetheless, other authors have also found differences between the masses derived from the APEX $870 \mu \mathrm{m}$ flux and the Herschel $\mathrm{H}_{2}$ column density, in the sense that $870 \mu \mathrm{m}$ masses are lower than $\mathrm{H}_{2}$ column density masses (Dehar- 
veng et al. 2010; Liu et al. 2017; Dewangan et al. 2017; Das et al. 2018). In particular, Liu et al. (2017) attributed this discrepancy to possible drawbacks in ground-based data reduction that underestimate the mass, due to the loss of large-scale emission when applying the sky noise subtraction. Hence, the mass derived from the $870 \mu \mathrm{m}$ flux may only represent a lower limit to the true mass. Moreover, the PPMAP procedure provides differential column density as a function of dust temperature and position (see Marsh et al. 2015). Consequently, hydrogen mass derived by the PPMAP method is probable more reliable and accurate than that calculated from the $870 \mu \mathrm{m}$ ATLASGAL flux density.

\section{THE DRIVING SOURCE}

Ogura (1993) proposed that the driving source of HH 137/138 should be located between HH 137-knot J and HH 138-knot A. The distance between the knots is about $1^{\prime}$. Bearing this in mind, we searched for all the young stellar objects (YSOs) that are likely to be the driving sources in an area of $60^{\prime \prime} \times 40^{\prime \prime}$. A number of YSOs were identified using the Spitzer and WISE point source catalogs. The Spitzer candidates were obtained from the MYStIX IR Excess Source catalog from Povich et al. (2013), who identified a few YSOs in the selected region. We have placed these YSOs in a Color-Color ([3.6]-[4.5] vs [5.8]-[8.0]) diagram and used the criteria by Allen et al. (2004) to determine their evolutionary status. The WISE candidates, in the same area, were obtained from Cutri \& et al. (2013) applying the criteria by Koenig et al. (2012). Class I candidates are protostars surrounded by dusty infalling envelopes, while Class II candidates are dominated by accretion disks. Both Class I as well as Class II young stars have been identified as the exciting sources of optical jets and molecular outflows (Bachiller \& Tafalla 1999; Reipurth \& Bally 2001; Arce et al. 2007).

Spitzer and WISE sources are labeled in Fig. 5, which is an overlay of the 4.5 (in green) and $8 \mu \mathrm{m}$ (in blue) Spitzer images, $160 \mu \mathrm{m}$ (in red contours) Herschel image and $4.6 \mu \mathrm{m}$ (in cyan contours) WISE image. Coordinates, fluxes in the four bands of each catalog, evolutionary status and cross-correlations of the Spitzer and WISE sources are listed in Table 3. All the WISE sources are also detected in the Spitzer catalog. The slight differences in position are attributed to the angular resolution of the corresponding instruments. Spitzer sources 1 and 2 (or WISE source 8) coincide with the peak of emission in Herschel-PACS $160 \mu \mathrm{m}$ identified as C1 (see Section 4) that also clearly shows up in $70 \mu \mathrm{m}$ (see Figure 3, lower panel). The coincidence of the emissions at 160 and $70 \mu \mathrm{m}$ with two YSO candidates suggests that dust temperature in this region is higher than in the rest of the dust clump, giving additional support to the YSO status of these sources. In addition, Walsh et al. (2014) found $\mathrm{H}_{2} \mathrm{O}$ maser emission coincident with Spitzer source 1 (see Table 3), thus reinforcing its YSO classification.

WISE source 8, which is likely to be associated with EGOs, is bright at $22 \mu \mathrm{m}$ (3.08 mag, see Table 3) and lies within the dark infrared cloud (see Section 4). According to the classification scheme of Chambers et al. (2009) it would be considered as an "active" core. WISE data at $22 \mu \mathrm{m}$ are being used instead of at $24 \mu \mathrm{m}$, as was originally proposed by Chambers et al. (2009), since Spitzer MIPS images at $24 \mu \mathrm{m}$ at the source position are not available.

The projected separation between Spitzer sources 1 and 2 is $5^{\prime \prime}$, or $\sim 7500 \mathrm{AU}$ (at $1.5 \mathrm{kpc}$ ). Young low mass binaries have separations from 500 to $4500 \mathrm{AU}$ (see Connelley et al. 2008 and references therein). Young massive binaries should expand a larger range of separations since the components are larger. Recently, Po-

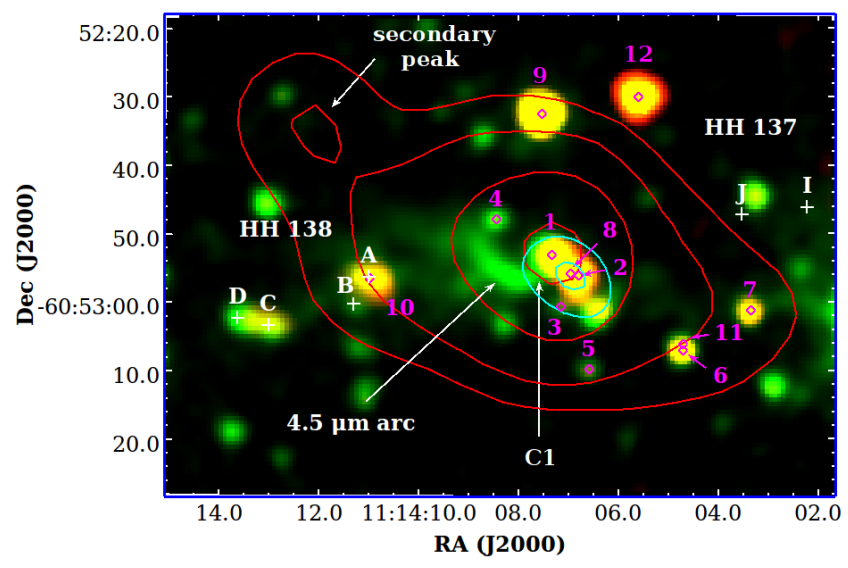

Figure 5. Overlay of the Spitzer 4.5 (in green) and $8 \mu \mathrm{m}$ (in red) images, and contours of Herschel $160 \mu \mathrm{m}$ (in red) and WISE $4.6 \mu \mathrm{m}$ (in cyan) images. The contours correspond to $0.05,0.1,0.3$ and $0.8 \mathrm{Jy} \mathrm{pixel}^{-1}$ for Herschel, and 0.05 and $0.07 \mathrm{Jy} \mathrm{pixel}^{-1}$ for WISE. The peak at $160 \mu \mathrm{m}$ is labeled as C1 at RA.,Dec. $(\mathrm{J} 2000)=(11: 14: 07.324,-60: 52: 52.90)$. A secondary weaker peak is also shown to the north-east at RA.,Dec. $(J 2000)=(11: 14: 12.054,-60: 52: 35.50)$. The pink diamonds and numbers correspond to the sources in Table 3 . The white crosses mark the optical knots of HH 137 and HH 138 from Ogura (1993) and the white arrows indicates the $4.5 \mu \mathrm{m}$ arc, the dust clump $\mathrm{C} 1$ and the secondary peak.

mohaci et al. (2019) performed a pilot survey of candidate wide young massive binary stars, finding that pairs (with a probability of chance alignment $<20 \%$ ) have separations between $\sim 1300$ and $\sim 13000$ UA. Thus, Spitzer sources 1 and 2 might be gravitationally bound, forming a wide binary system, assuming they are massive stars.

In summary, we propose that Spitzer sources 1 and 2, coinciding with WISE source 8 (WISE J111406.96-605255.9), are the candidate powering sources of the HH objects. Spitzer sources 1 and 2 are also very close to the $4.5 \mu \mathrm{m}$ arc-like structure and are projected onto the axis of HH 137. WISE source 8 has been also classified as YSO candidate in the AKARI/FIS young stellar objects catalog (Tóth et al. 2014).

\section{APEX MOLECULAR GAS RESULTS}

\subsection{Analysis of the $\mathrm{CO}$ data}

The ${ }^{12} \mathrm{CO}(3-2)$ line shows emission above $3 \sigma$ between $\approx-22$ and $+5 \mathrm{~km} \mathrm{~s}^{-1}$ (all velocities are referred to the LSR). The averaged spectra of the three $\mathrm{CO}$ isotopologues $\left({ }^{12} \mathrm{CO},{ }^{13} \mathrm{CO}\right.$ and $\left.\mathrm{C}^{18} \mathrm{O}\right)$, taken in an aperture of $132^{\prime \prime} \times 81^{\prime \prime}$ centered at RA.,Dec. $(\mathrm{J} 2000)=(11: 14: 06.497,-60: 52: 40.70)$ and covering most of the $\mathrm{CO}$ emission, are shown in Figure 6 . The ${ }^{12} \mathrm{CO}$ profile shows two peaks at -8.6 and $-5.2 \mathrm{~km} \mathrm{~s}^{-1}$, with a depression at $-7.1 \mathrm{~km} \mathrm{~s}^{-1}$. The blueshifted and redshifted wings are indicated with dashed line boxes and are associated with molecular outflows (see Section 7).

The ${ }^{13} \mathrm{CO}(3-2)$ line shows a shallower depression than the ${ }^{12} \mathrm{CO}(3-2)$ line profile centered at a similar velocity. The $\mathrm{C}^{18} \mathrm{O}(3-2)^{10}$ is centered at $-6.3 \mathrm{~km} \mathrm{~s}^{-1}$, redshifted with respect

${ }^{10} \mathrm{C}^{18} \mathrm{O}$ is a high-density molecular tracer with a critical density of $\sim 1.2 \times 10^{4} \mathrm{~cm}^{-3}$ in $\mathrm{J}=3-2$ estimated from Shirley (2015), assuming a kinetic temperature of $\sim 15 \mathrm{~K}$. 
Table 3. YSO candidates projected onto the central region of HH 137/138.

\begin{tabular}{|c|c|c|c|c|c|c|c|c|c|}
\hline \multicolumn{10}{|c|}{ Spitzer candidates } \\
\hline \multirow[t]{2}{*}{$\#$} & \multirow{2}{*}{$\begin{array}{l}\text { R.A. } \\
(h: m: s)\end{array}$} & \multirow{2}{*}{$\begin{array}{l}\text { Dec. } \\
\left({ }^{\circ}:^{\prime}:^{\prime \prime}\right)\end{array}$} & \multirow{2}{*}{$\begin{array}{l}\text { Spitzer } \\
\text { name }\end{array}$} & \multicolumn{4}{|c|}{ Fluxes [mag] } & \multirow[t]{2}{*}{ YSO Class } & \multirow{2}{*}{$\begin{array}{c}\text { Ref. WISE } \\
\#\end{array}$} \\
\hline & & & & {$[3.6]$} & {$[4.5]$} & {$[5.8]$} & {$[8.0]$} & & \\
\hline 1 & $11: 14: 07.33$ & $-60: 52: 53.25$ & G291.3671-00.2137 & 10.569 & 9.877 & 9.300 & 8.599 & I & 8 \\
\hline 2 & $11: 14: 06.80$ & $-60: 52: 56.12$ & G291.3664-00.2148 & 12.533 & 11.111 & 10.027 & 9.155 & I & 8 \\
\hline 3 & $11: 14: 07.15$ & $-60: 53: 00.75$ & G291.3671-00.2157 & 14.760 & 13.516 & 12.933 & 11.895 & I & - \\
\hline 4 & 11:14:08.44 & $-60: 52: 47.97$ & G291.3687-00.2115 & 13.092 & 12.497 & 11.986 & 11.349 & II & - \\
\hline 5 & $11: 14: 06.58$ & $-60: 53: 09.82$ & G291.3674-00.2185 & 14.004 & 13.088 & 12.500 & 11.592 & I & - \\
\hline 6 & 11:14:04.70 & $-60: 53: 07.05$ & G291.3635-00.2192 & 12.396 & 11.604 & 10.922 & 10.174 & II & 11 \\
\hline 7 & $11: 14: 03.35$ & $-60: 53: 01.31$ & G291.3604-00.2187 & 12.874 & 11.931 & 11.154 & 10.411 & II & - \\
\hline \multicolumn{10}{|c|}{ WISE candidates } \\
\hline \multirow[t]{2}{*}{$\#$} & \multirow{2}{*}{$\begin{array}{c}\text { R.A. } \\
(h: m \cdot s)\end{array}$} & \multirow{2}{*}{$\begin{array}{l}\text { Dec. } \\
\left({ }^{\circ}:^{\prime}:^{\prime \prime}\right)\end{array}$} & \multirow{2}{*}{$\begin{array}{l}\text { WISE } \\
\text { name }\end{array}$} & \multicolumn{4}{|c|}{ Fluxes [mag] } & YSO Class & Ref. Spitzer \\
\hline & & & & {$[3.4]$} & {$[4.6]$} & {$[12.0]$} & {$[22.0]$} & & \# \\
\hline 8 & $11: 14: 06.96$ & $-60: 52: 55.96$ & J111406.96-605255.9 & 12.176 & 9.653 & 8.106 & 3.08 & II & $1-2$ \\
\hline 9 & $11: 14: 07.53$ & $-60: 52: 32.57$ & $\mathrm{~J} 111407.53-605232.5$ & 9,849 & 9.155 & 9.78 & 6.882 & II & - \\
\hline 10 & $11: 14: 10.98$ & $-60: 52: 56.53$ & $\mathrm{~J} 111410.98-605256.5$ & 13.065 & 10.973 & 10.278 & 6.242 & II & - \\
\hline 11 & 11:14:04.70 & $-60: 53: 06.25$ & $\mathrm{~J} 111404.69-605306.2$ & 13.144 & 11.365 & 10.274 & 6.506 & II & 6 \\
\hline 12 & 11:14:05.59 & $-60: 52: 30.08$ & $\mathrm{~J} 111405.58-605230.0$ & 11.759 & 10.589 & 7.90 & 5.081 & $\mathrm{I}$ & - \\
\hline
\end{tabular}

to the ${ }^{12} \mathrm{CO}$ depression. As expected, the ${ }^{13} \mathrm{CO}$ and $\mathrm{C}^{18} \mathrm{O}$ profiles are narrower (in velocity) and weaker (in $\mathrm{T}_{m b}$ ) than the ${ }^{12} \mathrm{CO}$ profile and have emissions within the velocity intervals $[-9.5,-3.6] \mathrm{km} \mathrm{s}^{-1}$, and $[-8,-4] \mathrm{km} \mathrm{s}^{-1}$, respectively.

Taking into account the Galactic non-circular motions (Brand $\&$ Blitz 1993) and a systemic velocity of $-6.3 \mathrm{~km} \mathrm{~s}^{-1}$ (obtained from the $\mathrm{C}^{18} \mathrm{O}$ peak) with an uncertainty of $5 \mathrm{~km} \mathrm{~s}^{-1}$, we estimated the kinematic distance. As the Carina region is located in the fourth quadrant at the inner Galaxy, there are two kinematic distances along the line of sight: the "near" and "far". Since the region has a high extinction (see Figure 1) and has been catalogued as an IRDC (Simon et al. 2006b; Jackson et al. 2008; Dobashi 2011), HH 137 and HH 138 should lie in front of the Galaxy and near the solar circle. Then, we adopt the "near" kinematic distance that turns out to be equal to $1.5 \pm 0.5 \mathrm{kpc}$. This value is compatible with those calculated by Kavars et al. (2005); Jackson et al. (2008); Barnes et al. (2011); Planck Collaboration et al. (2016) and roughly agrees with the distance adopted by Ogura (1993) of $2.2 \pm 0.2 \mathrm{kpc}$.

Figures 7 and 8 display the ${ }^{12} \mathrm{CO},{ }^{13} \mathrm{CO}$ and $\mathrm{C}^{18} \mathrm{O}$ integrated emissions at different velocity ranges overlaid on a three-color combined image: Gemini $\mathrm{K}$ (blue) and $\mathrm{H}_{2}$ (green) and Spitzer $4.5 \mu \mathrm{m}$ (red). The dashed green line box marks the area observed with Gemini. In particular, the ${ }^{12} \mathrm{CO}$ molecule in Figure 7 shows an extended distribution with multiple peak emissions mostly concentrated towards the knots of $\mathrm{HH} 137$ and $\mathrm{HH}$ 138. It is interesting to note that the position of one of the $\mathrm{CO}$ emission peaks, showing an elongated shape (named B2), matches the alignment of all $\mathrm{HH} 137$ knots, including the $\mathrm{H}_{2}$ terminal bow shock (see panel $d$ )). In Section 7, we analyze such alignment in more detail.

Figure 8 shows the ${ }^{13} \mathrm{CO}$ and $\mathrm{C}^{18} \mathrm{O}$ integrated emission between -9 and $-3 \mathrm{~km} \mathrm{~s}^{-1}$ in step of $2 \mathrm{~km} \mathrm{~s}^{-1}$. In this velocity range, we find that the molecular distribution would be associated to a single cloud with an oblate shape orientated from the north-east to the south-west. Since these molecules trace higher densities than the ${ }^{12} \mathrm{CO}$, this single cloud will be refereed as a dense clump, coinciding with the dust clump $\mathrm{C} 1$ in Figure 5. Nonetheless, when inspecting the ${ }^{13} \mathrm{CO}$ distribution in more detail (see Figure 8, panel $b)$ ), we detect the molecular counterpart of the secondary dust peak shown in Figure 5 , detected at $\lambda \geq 160 \mu \mathrm{m}$. This second peak is observed between -6.0 and $-4.5 \mathrm{~km} \mathrm{~s}^{-1}$. However, we are not able to completely resolve the two ${ }^{13} \mathrm{CO}$ peaks as different components due to the low resolution of the APEX data.

Fitting an ellipse to the $2.5 \mathrm{~K}$ contour $(5 \sigma)$ of the ${ }^{13} \mathrm{CO}$ integrated emission (between $[-9.5,-3.6] \mathrm{km} \mathrm{s}^{-1}$ ), we estimate major and minor semi-axes of the molecular clump C1 of $69.6^{\prime \prime} \times 40.3^{\prime \prime}$ $\left(\sim 0.51 \times 0.29 \mathrm{pc}\right.$, with a P.A. of $\left.20^{\circ}\right)$, giving a deconvolved, effective radius $R_{\text {eff }}=0.38 \mathrm{pc}$. The center of the fitted ellipse ( $\alpha=11: 14: 09.2, \delta=-60: 52: 42.700)$ is located $7^{\prime \prime}$ to the north of the maximum emission in ${ }^{13} \mathrm{CO}$, and at $20^{\prime \prime}$ to the north-east of the suggested exciting source (source 8 in Table 3). However, these angular separations are practically coincident with the APEX spatial resolution. On the other hand, the $\mathrm{C}^{18} \mathrm{O}$ integrated emission shows a small condensation only between -7 and $-5 \mathrm{~km} \mathrm{~s}^{-1}$ surrounded by the ${ }^{13} \mathrm{CO}$ emission.

\section{2 $\mathrm{HCO}^{+}(3-2)$ and $\mathrm{HCN}(3-2)$ data}

Figure 9 shows averaged profiles of $\mathrm{HCO}^{+}(3-2)$ (upper panel) and of $\mathrm{HCN}(3-2)$ (lower panel) within the emitting region of clump $\mathrm{C} 1$. Critical densities for these lines and transitions are: $\sim 1.5 \times 10^{6} \mathrm{~cm}^{-3}$ and $\sim 1.2 \times 10^{7} \mathrm{~cm}^{-3}, \mathrm{HCO}^{+}(3-2)$ and $\mathrm{HCN}(3-2)$ respectively, for a kinetic temperature $\mathrm{T}_{k} \sim 15 \mathrm{~K}$ (Shirley 2015). Thus, the detection of these lines towards clump $\mathrm{C} 1$ indicates ambient densities compatible with these values in the central part of the clump. The $\mathrm{HCO}^{+}$line has a peak at $-5.8 \mathrm{~km} \mathrm{~s}^{-1}$ and a weak absorption at $-6.8 \mathrm{~km} \mathrm{~s}^{-1}$. The HCN line shows a peak at $-5.7 \mathrm{~km} \mathrm{~s}^{-1}$ and an absorption, deeper than the $\mathrm{HCO}^{+}$profile, at $-6.8 \mathrm{~km} \mathrm{~s}^{-1}$. In addition, the $\mathrm{HCN}$ average line profile is wider than the $\mathrm{HCO}^{+}$ profile (6 vs $3 \mathrm{~km} \mathrm{~s}^{-1}$ at FWHM).

Figure 10 shows the spatial distribution of the $\mathrm{HCO}^{+}$and $\mathrm{HCN}$ emissions overlaid with the IRAC/Spitzer 3.6, 4.5 and $8.0 \mu \mathrm{m}$ images. In $\mathrm{HCO}^{+}$, the elongated emitting region is $56^{\prime \prime} \times 30^{\prime \prime}(0.41 \mathrm{pc} \times 0.22 \mathrm{pc})$, and it is detected in the interval $[-11.8,-2.9] \mathrm{km} \mathrm{s}^{-1}$. In the case of $\mathrm{HCN}$, the emission is present 


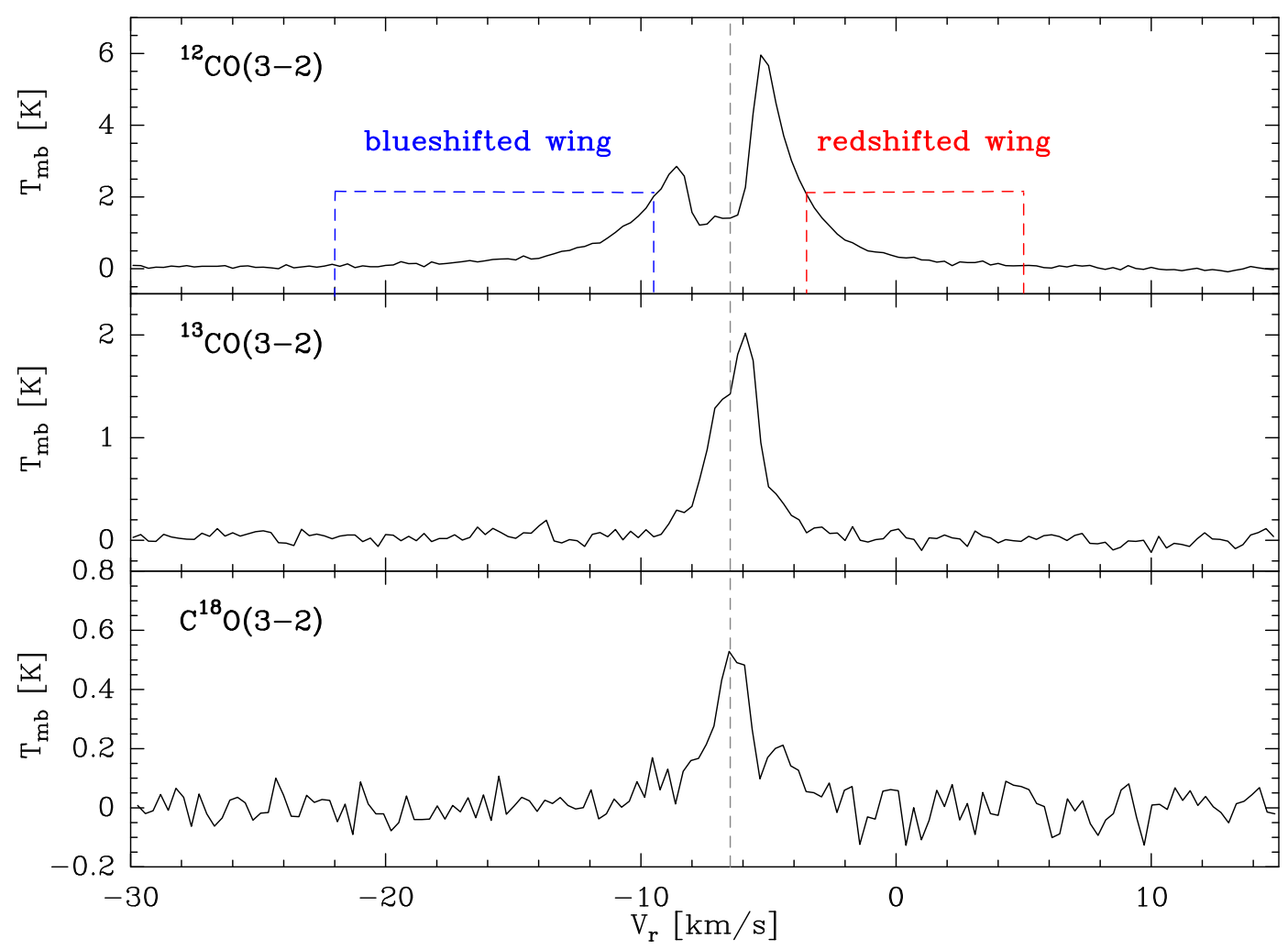

Figure 6. Averaged profiles of ${ }^{12} \mathrm{CO}(3-2),{ }^{13} \mathrm{CO}(3-2)$ and $\mathrm{C}^{18} \mathrm{O}(3-2)$ within the emitting region in the velocity interval $[-30,+15] \mathrm{km} \mathrm{s}^{-1}$ showing main-beam brightness temperature $\mathrm{T}_{m b}$ vs. LSR velocity. The vertical dashed gray line indicates the velocity position corresponding to the emission peak of $\mathrm{C}^{18} \mathrm{O}(3-2)$ taken as the systemic velocity. The dashed blue and red line boxes in the upper panel show the ${ }^{12} \mathrm{CO}(3-2)$ wings of molecular outflows (see Section 7).

in a region of $42^{\prime \prime} \times 21^{\prime \prime}(0.31 \mathrm{pc} \times 0.15 \mathrm{pc})$ in the velocity range $[-12.3,0.4] \mathrm{km} \mathrm{s}^{-1}$. These sizes are estimated from contours at $5 \sigma$ in the integrated emission. In both lines, the emission coincides with the brightest part of $\mathrm{C} 1$ and the cold dust emission (see Figure 8). In particular, the arc at $4.5 \mu \mathrm{m}$ is projected onto the brightest regions, while knots $\mathrm{A}$ and $\mathrm{B}$ belonging to $\mathrm{HH} 138$ are within the molecular region as well as the proposed driving source (see upper panel of Figure 3).

\subsection{Physical parameters of the molecular core}

We estimated the $\mathrm{H}_{2}$ mass of clump $\mathrm{C} 1$ assuming LTE condition through both ${ }^{13} \mathrm{CO}$ and $\mathrm{C}^{18} \mathrm{O}$ emissions. We assumed that the excitation temperature is the same for both molecules with a value of $\mathrm{T}_{\text {exc }}=15 \mathrm{~K}$. To fix this value, we calculated the excitation temperature from the ${ }^{12} \mathrm{CO}$ maximum giving $\mathrm{T}_{\text {exc }}=13.4 \mathrm{~K}$, close to the dust temperature $\mathrm{T}_{\text {dust }}=14.8 \mathrm{~K}$ (see Section 4). Nevertheless, the derivation of $\mathrm{T}_{\mathrm{exc}}$ from the ${ }^{12} \mathrm{CO}$ maximum was not completely reliable due to the self-absorption of this line (see Figure 6, upper panel). However, this $\mathrm{T}_{\text {exc }}$ should not be too high since the characteristic excitation temperature for IRDCs is $\sim 10 \mathrm{~K}$ (see Du \& Yang 2008). Thus, we assumed for this region an excitation temperature close to the dust temperature of $15 \mathrm{~K}$.

We followed the equations of Bourke et al. (1997) to derive the optical depths $\left(\tau_{13}, \tau_{18}\right)$, column densities and the molecular mass. The optical depths of ${ }^{13} \mathrm{CO}$ and $\mathrm{C}^{18} \mathrm{O}$ were estimated using the following equation:

$\tau=-\ln \left[1-\frac{T_{\mathrm{peak}}}{T^{*}}\left[\left(e^{\frac{T^{*}}{T_{\mathrm{exc}}}}-1\right)^{-1}-\left(e^{\frac{T^{*}}{T_{\mathrm{bg}}}}-1\right)^{-1}\right]^{-1}\right]$,

where, $T^{*}=h v / k$, with $v$ the rest frequency of the ${ }^{13} \mathrm{CO}(3-2)$ and $\mathrm{C}^{18} \mathrm{O}(3-2)$ lines and $T_{\text {bg }}$ the background temperature of $2.7 \mathrm{~K}$. To obtain the main-beam brightness temperature peaks for both molecular lines $\left(T_{\text {peak }}^{13}, T_{\text {peak }}^{18}\right)$, we performed a Gaussian fit to the ${ }^{13} \mathrm{CO}$ and $\mathrm{C}^{18} \mathrm{O}$ averaged spectra (Figure 6) within the area of the molecular clump $\left(132^{\prime \prime} \times 81^{\prime \prime}\right.$, with a P.A. of $\left.20^{\circ}\right)$. The ${ }^{13} \mathrm{CO}$ and $\mathrm{C}^{18} \mathrm{O}$ molecular lines have peak temperatures of $\sim 2.0$ and $\sim 0.55 \mathrm{~K}$, respectively. However, since the ${ }^{13} \mathrm{CO}$ profile is weakly affected by a self-absorption (see Figure 6 , middle panel), we adopted a $T_{\text {peak }}^{13} \approx 2.5 \mathrm{~K}$.

With these peak temperatures, we estimated that $\tau_{13}$ and $\tau_{18}$ are 0.33 and 0.07 , respectively, showing that both lines are optically thin. Then, the ${ }^{13} \mathrm{CO}$ column density was estimated using:

$N\left({ }^{13} \mathrm{CO}\right)=8.07 \times 10^{13} e^{\frac{15.86}{T_{\mathrm{exc}}}}\left[\frac{T_{\mathrm{exc}}+0.88}{1-e^{-\frac{15.86}{T_{\mathrm{exc}}}}}\right] \int \tau^{13} d v\left(\mathrm{~cm}^{-2}\right)$.

Since the ${ }^{13} \mathrm{CO}$ line is optically thin, the integral of Eq. 3 can be replaced by

$\int \tau^{13} d v \approx \frac{1}{J\left(T_{\mathrm{ex}}\right)-J\left(T_{\mathrm{bg}}\right)} \int T_{\mathrm{mb}} d v$, 

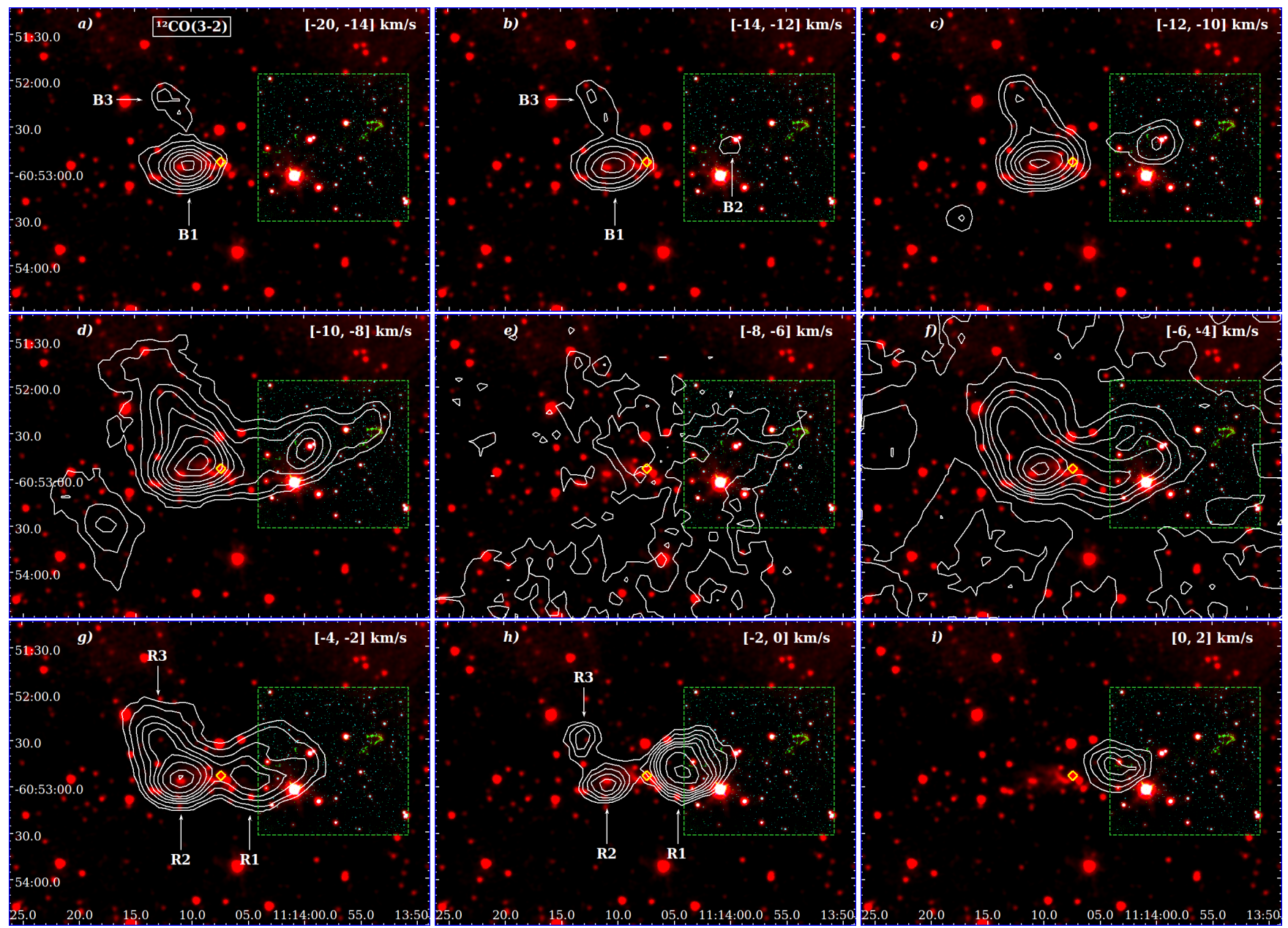

Figure 7. ${ }^{12} \mathrm{CO}(3-2)$ line emissions in the velocity intervals indicated in the upper right corner of each image, superimposed on a combined three-band images: $\mathrm{H}_{2}$ (in green), $\mathrm{K}$ (in blue) and $4.5 \mu \mathrm{m}$ (in red). All panel have a velocity range of $2 \mathrm{~km} \mathrm{~s}^{-1}$ except the panel $a$ ), which interval is $6 \mathrm{~km} \mathrm{~s}{ }^{-1}$ wide. ${ }^{12} \mathrm{CO}(3-2)$ white contours of panels $a$ ) to $d$ ), $f$ ) and $g$ ) correspond to $2,3,5,7,9,11,14,18,21$ and $21 \mathrm{~K} \mathrm{~km} \mathrm{~s}^{-1}$. Panel $e$ ) shows white contours at 3.5 and $4 \mathrm{~K} \mathrm{~km} \mathrm{~s}^{-1}$. Panel $h$ ) and $i$ ) display white contours at 1.5, 2, 2.5, 3, 3.5, 4, 5 and $6 \mathrm{~K} \mathrm{~km} \mathrm{~s}^{-1}$. The dashed green line box shows the area observed with Gemini. The yellow diamond indicates the location of the suggested exciting source (see Section 5). In panels $a$ ), b), g) and $h$ ) blueshifted (B1, B2 and B3) and redshifted (R1, R2 and R3) structures are marked.

with

$J(T)=\frac{T^{*}}{e^{\frac{T^{*}}{T_{\mathrm{ex}}}}-1}$.

The total molecular hydrogen mass is calculated using:

$\mathrm{M}\left(\mathrm{H}_{2}\right)=\mu m_{H} A N\left(\mathrm{H}_{2}\right) d^{2}$

$\left(M_{\odot}\right)$

where $\mu$ is the mean molecular weight, equal to 2.76 for a relative helium abundance of $25 \%$ by mass (Yamaguchi et al. 1999), $\mathrm{m}_{\mathrm{H}}$ is the hydrogen atom mass $\left(1.67 \times 10^{-24} \mathrm{~g}\right), A$ is the area subtended by the ${ }^{13} \mathrm{CO}$ clump, $\mathrm{N}\left(\mathrm{H}_{2}\right)$ the total molecular column density, and $d$ is the adopted distance $(1.5 \pm 0.5 \mathrm{kpc})$. To obtain the $\mathrm{N}\left(\mathrm{H}_{2}\right)$ and the total molecular hydrogen mass, an abundance of $\left[\mathrm{H}_{2} /{ }^{13} \mathrm{CO}\right]=1 \times 10^{6}$ (Saldaño et al. 2019) was adopted. Our result for the clump $\mathrm{C} 1$ turns out to be $\mathrm{N}\left(\mathrm{H}_{2}\right)=4.3 \times 10^{21} \mathrm{~cm}^{-3}$, and $\mathrm{M}\left(\mathrm{H}_{2}\right)=36 \mathrm{M}_{\odot}$, within an area with an effective radius of $R_{\text {eff }}=0.38$ pc. The uncertainty in the column density is about $20 \%$. Such error originates mainly in the inaccuracy of the distance. In addition, the unknown geometry of the source and the uncertainty in abundances also contribute to the estimated error.
With regard to the mass, if we considered $\mathrm{T}_{\mathrm{exc}}=15 \pm 5 \mathrm{~K}$, the mass would turn out to be $\mathrm{M}\left(\mathrm{H}_{2}\right)=36 \pm 29 \mathrm{M}_{\odot}$.

\section{THE MOLECULAR OUTFLOWS}

As mentioned in Section 6.1, the ${ }^{12} \mathrm{CO}(3-2)$ profile shows noticeable wings at lower and higher velocities with respect to the systematic velocity. We applied the criterion of Hatchell et al. $(2007)^{11}$ to identify both redshifted and blueshifted components (red lobes and blue lobes hereafter) between $[-20,-9.3] \mathrm{km} \mathrm{s}^{-1}$ and $[-3.3,+5] \mathrm{km} \mathrm{s}^{-1}$, respectively. These velocity ranges are indicated with dashed red and blue line boxes in Figure 6 .

Figure 11, upper panel, shows the ${ }^{12} \mathrm{CO}$ line-wings integrated emission overlaid on a combined three-color image, $\mathrm{K}$ (blue), $\mathrm{H}_{2}$ (green) and $4.5 \mu \mathrm{m}$ (red), showing that four lobes (two red and

11 These authors provide an objective criterion to identify sources with outflows by measuring the ${ }^{12} \mathrm{CO}(3-2)$ line-wings with more than $3 \sigma$ at $3 \mathrm{~km} \mathrm{~s}^{-1}$ from the $\mathrm{C}^{18} \mathrm{O}(3-2)$ line center. 

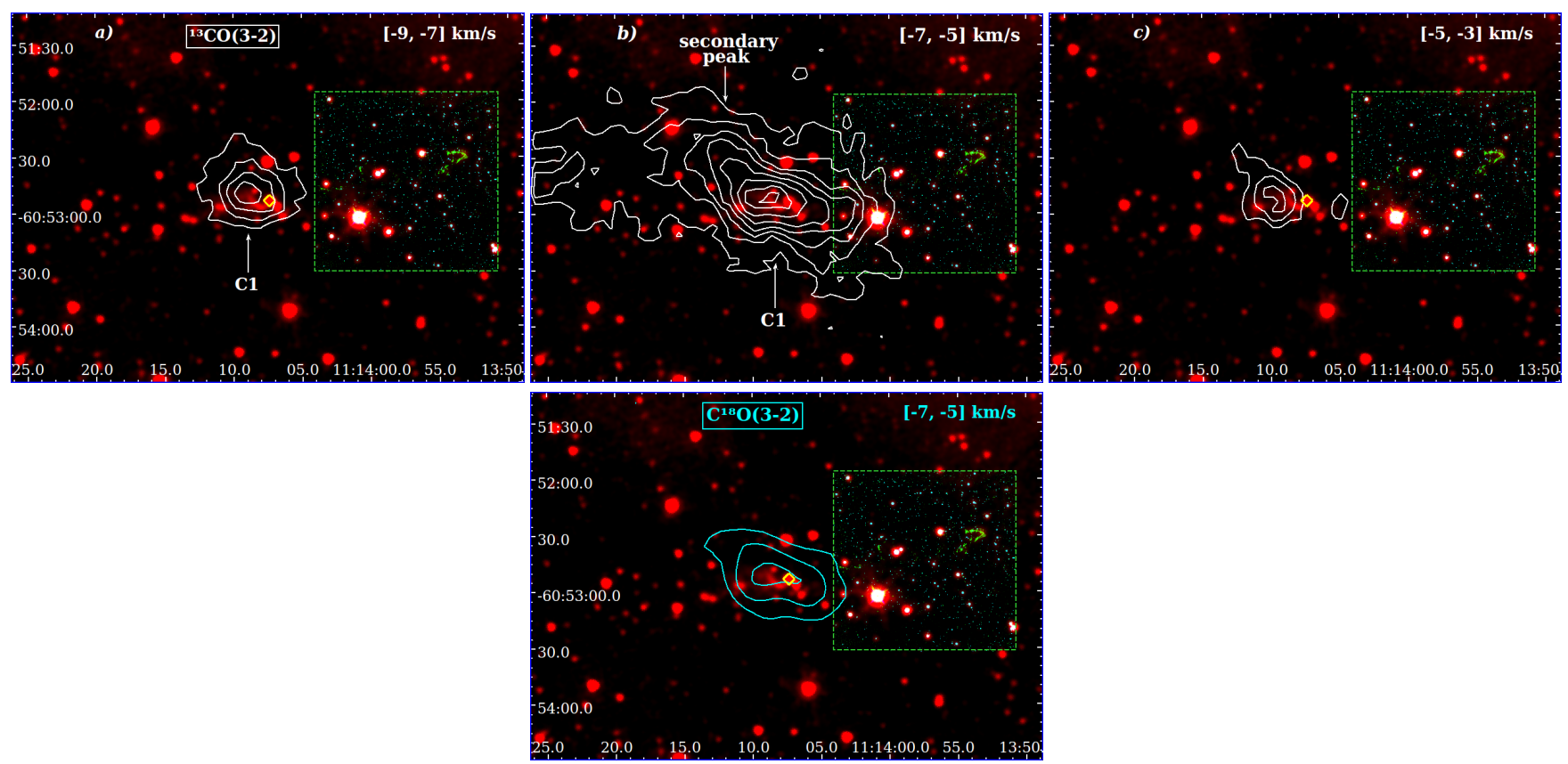

Figure 8. ${ }^{13} \mathrm{CO}(3-2)$ and $\mathrm{C}^{18} \mathrm{O}(3-2)$ line emissions in the velocity intervals indicated in the upper right corner of each image, superimposed on a combined three-band images: $\mathrm{H}_{2}$ (in green), $\mathrm{K}$ (in blue) and $4.5 \mu \mathrm{m}$ (in red). All panel have a velocity range of $2 \mathrm{~km} \mathrm{~s}^{-1} \cdot{ }^{13} \mathrm{CO}(3-2)$ white contours (upper panels from a) to $c$ ) ) correspond to $2,3,4,5,6,7,8$ and $8.8 \mathrm{~K} \mathrm{~km} \mathrm{~s}^{-1}$. The lower panel shows $\mathrm{C}^{18} \mathrm{O}(3-2)$ cyan contours at $1,1.5$ and $2 \mathrm{~K} \mathrm{~km} \mathrm{~s}^{-1}$. Dense gas coinciding with the positions of the dust clump $\mathrm{C} 1$ and a secondary dust peak in Figure 5 is indicated. The dashed green line box shows the area observed with Gemini. The yellow diamond indicates the location of the suggested exciting source (see Sec. 5).

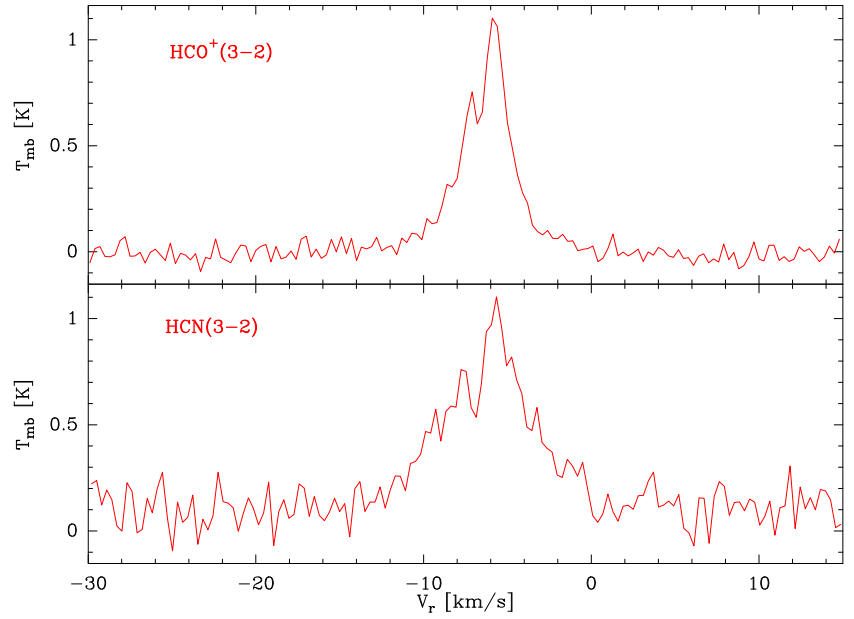

Figure 9. Averaged profiles of $\mathrm{HCO}^{+}(3-2)$ and $\mathrm{HCN}(3-2)$ within the emitting region of clump $\mathrm{C} 1$ in the velocity interval $[-30,+15] \mathrm{km} \mathrm{s}^{-1}$ showing main-beam brightness temperature $\mathrm{T}_{m b}$ vs. LSR velocity.

two blue) are aligned with the knots of HH 137 and HH 138. Performing a cut along the knots direction, as is indicated in the upper panel of Figure 11 with a double arrow dashed yellow line, two blue lobes (named B1 and B2) and two red lobes (R1 and R2) are identified in the position-velocity (PV) diagram of Figure 12 . The cut goes from RA.,Dec. $($ J2000 $)=(11: 13: 49.605,-60: 52: 21.69)$ to (11:14:20.693, -60:53:13.05), with offset coordinates centered on the proposed exciting source position indicated with a horizontal dashed white line in this figure (see Section 5). Panels a) and b) of Figure 11 show ${ }^{12} \mathrm{CO}$ contours emissions for these two outflows (outflows 1 and 2).

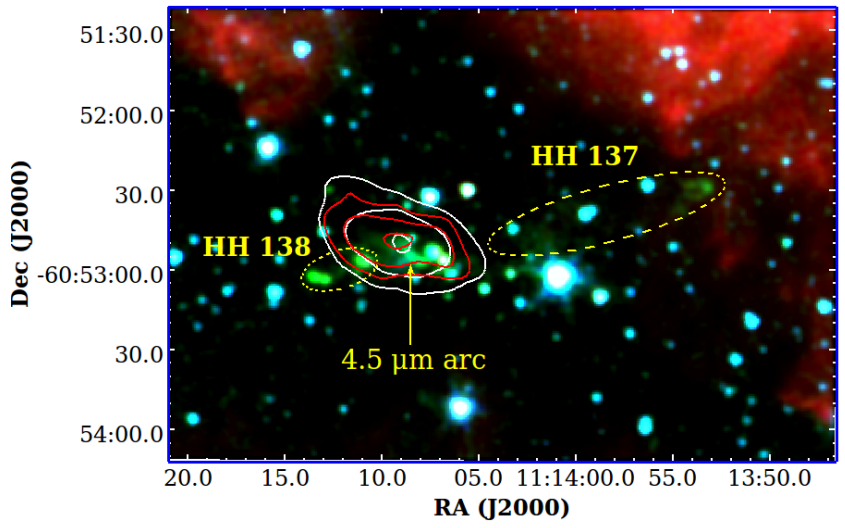

Figure 10. Spatial distribution of $\mathrm{HCO}^{+}$and $\mathrm{HCN}$ emissions overlaid with the Spitzer/IRAC images. The white contours are from $\mathrm{HCO}^{+}(3-2)$ and correspond to $0.7,1.2$ and $1.7 \mathrm{~K}$. The red contours are from $\mathrm{HCN}(3-2)$ and correspond to $0.5,0.7$ and $0.9 \mathrm{~K}$. The combined IRAC images are $3.6 \mu \mathrm{m}$ (in blue), $4.5 \mu \mathrm{m}$ (in green) and $8.0 \mu \mathrm{m}$ (in red). The dashed yellow ellipses show the location of $\mathrm{HH} 137$ and $\mathrm{HH} 138$, and the yellow arrow indicates the $4.5 \mu \mathrm{m}$ arc.

The B1 lobe, as is shown in Figures 11 (middle panel a)) and 12 (upper panel), is observed from -22 to $-9.3 \mathrm{~km} \mathrm{~s}^{-1}$, while the $\mathrm{B} 2$ lobe appears from $-14 \mathrm{~km} \mathrm{~s}^{-1}$. The elongated shape of B2, which coincides with all $\mathrm{H}_{2}$ knots of $\mathrm{HH} 137$, ends at the terminal bow shock to the north-west. Meanwhile, B1 is located towards Ogura (1993)'s knots of HH 138, as well as superimposed to the $\mathrm{R} 2$ red lobe. This later lobe is less extended in velocity (from -3.3 to $0 \mathrm{~km} \mathrm{~s}^{-1}$ ). On the other hand, the $\mathrm{R} 1$ red lobe is more extended in velocity than $\mathrm{R} 2$ (from -3.3 to $+5 \mathrm{~km} \mathrm{~s}^{-1}$ ) and overlays with the knots of HH 137 that lie closer the proposed exciting source 
(see Section 5). Asymmetries in the PV diagram similar to those in Figure 12, upper panel, have been previously observed in other bipolar outflows analyzed using data of similar spatial resolution to the APEX data (e.g., Bourke et al. 1997; Sanhueza et al. 2010). In view of this, we associate the B1 blue lobe with the R1 red lobe, and similarly the B2 lobe to the R2 lobe. These associations are indicated in panels a) and b) of Figure 11.

Our description of the blueshifted and redshifted lobes is consistent with a scenario in which the driving sources, nested in the dense clump $\mathrm{C} 1$, develop two molecular outflows projected along the $\mathrm{H}_{2}$ knots. As was shown in Figure 5, at least two Spitzer Class I sources (YSO 1 and 2 in Table 3) lying towards the dust clump were found. As discussed in Section 5, YSO 1 and 2 have a projected separation of $\sim 7500 \mathrm{AU}$ and might form a wide binary system, being each star the exciting source of each outflow.

Multiple bipolar molecular outflows are commonly associated with multiple protostellar systems sharing the same parental envelope (e.g., Wu et al. 2009; Lee et al. 2016). In addition, the orientations of the outflows axes may provide some hint on the directions of multiple protostellar rotation or spin axes. Different proposed scenarios for the formation of aligned binary or multiple systems have been enumerated by Lee et al. (2016). These scenarios foresee the following: a) formation in a massive disk or ring in which two or more large co-rotating structures appear, b) binary components born by fragmentation of a core which originates two or more central objects with aligned angular momentum vectors, c) tidal effects that bring together two or more protostellar objects during subsequent evolutionary phases.

In the case of the outflows in Figures 12, panels a) and b), the inclination angles with respect to the plane of the sky should be small since both the red and blue lobes are well resolved and the centers of each lobe pair are separated by $\sim 47^{\prime \prime}$ (outflow 1 ) and $81^{\prime \prime}$ (outflow 2), higher than APEX HPBW of $\sim 20^{\prime \prime}$. These outflows show similar P.A. (with an angular difference of about $\sim 8^{\circ}$ ). Nonetheless, the projected angular moments of both outflows are probably in opposite senses. Hence, assuming that these outflows are associated with a binary system, the exciting stars would have almost aligned but opposite direction rotation axes, which may favor the later tidal scenario for the binary pair formation.

Figure 13 shows a schematic representation of the positions of outflows 1 and 2 with respect to the plane of the sky. In the left panel, the outflows are at the same distance and the driving sources form a binary star whereas in the right panel, the outflows are at different distances and no physical association between the exciting sources exits. Thus, the outflows are seen projected on the plane of the sky. Observations at higher spatial resolution, like those that can be performed by the Atacama Large Millimeter/submillimeter Array (ALMA) telescope, are needed to shed light on the outflows alignment and the binary nature of the exciting source.

On the other hand, a third likely weak outflow may be identified to the north-east of the four-lobe system (outflows 1 and 2). The blue and red lobes, named as B3 and R3 in the panel c) of Figure 11, are observed between $[-16.7,-9.3] \mathrm{km} \mathrm{s}^{-1}$ and $[-3.3,0.0] \mathrm{km} \mathrm{s}^{-1}$, respectively. These lobes are projected towards the weaker secondary peak, observed at wavelength $\geq 160 \mu \mathrm{m}$ (see Figure 5). Figure 12, lower panel, shows the PV diagram of outflow 3. The cut along this outflow is indicated with double arrow dashed cyan line in Figure 11 and goes from RA.,Dec. $($ J2000 $)=(11: 14: 21.222,-60: 53: 46.48)$ to (11:14:06.512, -60:51:23.76). Offset positions are centered 10.8" to the north-west of the secondary peak and indicated with a horizontal dashed white line in the lower panel of Figure 12. If the third outflow was associated with the secondary peak, this condensed structure would be a very young object surrounded by a cold and thick envelope completely shielding the exciting protostar. The lack of WISE and Spitzer young candidate sources near the secondary peak (see Figure 5) might provide support to this suggestion. Figure 12, lower panel, also shows blueshifted emission identified as B*, corresponding to the blue contours at the southeast corner in Figure 11.

Several physical parameters of the outflows can be estimated following, for example, Beuther et al. (2002); Yang et al. (2018) and de Villiers et al. (2014). They provide information about energy and mass that help to characterize the outflow. The parameters obtained from the molecular observations are: the length $r$ of the jet, the integrated emission in ${ }^{12} \mathrm{CO}(3-2)$ along the velocity range, the mass of the outflow $M_{\text {out }}$, the momentum $p$, the mechanical energy $E$, the time scale $t$, the mass entertainment rate $\dot{M}_{\text {out }}$, the mechanical force $F_{m}$, and the mechanical luminosity $L_{m}$ :

$$
\begin{aligned}
p & =M_{\text {out }} \times \mathrm{v}_{\text {max }- \text { out }}, \\
E & =\frac{1}{2} M_{\text {out }} \times \mathrm{v}_{\text {max }- \text { out }}^{2}, \\
t & =\frac{r}{\mathrm{v}_{\text {max }- \text { out }}}, \\
\dot{M}_{\text {out }} & =\frac{M_{\text {out }}}{t}, \\
F_{m} & =\frac{p}{t}, \\
L_{m} & =\frac{E}{t},
\end{aligned}
$$

where $\mathrm{v}_{\max }$-out is the maximum velocity in the blue and red lobes, respectively. Bearing in mind that the whole outflows are detected in the ${ }^{12} \mathrm{CO}(3-2)$ line only (with exception of a small core, which is seen in the ${ }^{13} \mathrm{CO}(3-2)$ and $\mathrm{C}^{18} \mathrm{O}$ lines), we estimated the $\mathrm{H}_{2}$ mass using the relation between the $\mathrm{H}_{2}$ column density and the ${ }^{12} \mathrm{CO}$ integrated emission. Taking into account the solid angle of the outflows (which give the linear sizes, $r$, in Table 4), the corresponding $\mathrm{H}_{2}$ masses amount to $M_{\text {out }}=17,5$ and $4 \mathrm{M}_{\odot}$, outflows 1,2 and 3 .

Table 4 lists these parameters for the three outflows in Figures 11 and 12. The values given in this table are not corrected by the unknown inclination angle. However, as mentioned before in this section, in the cases of outflows 1 and 2, red and blue lobes are well resolved and the corresponding centers have separations larger than APEX data HPBW, which indicates that the inclination angles should be small. The length of the outflow 2 from the terminal bow shock to the proposed driving source is $0.9 \pm 0.3 \mathrm{pc}$. However, this parameter depends on the inclination of the outflow with respect to the plane of the sky.

Downes \& Cabrit (2007) suggest that no inclination correction is needed for the momentum, since, by coincidence, underestimates along the jet axis are canceled by overestimates in the transverse component. On the other hand, the energy is overestimated by the inclination angle. Correction factors propose by these authors are applied to jet driven outflows emanating from Class 0 sources than have not yet broken out the parent cloud, which is not the case of outflows 1 and 2 .

The parameters of the outflows identified in the HH 137 and HH 138 region are, in general, quite similar to those reported by Beuther et al. (2002) in high-mass star-forming regions and by Yang et al. (2018) towards ATLASGAL clumps. The first authors used the ${ }^{12} \mathrm{CO} J=2-1$ transition to identified outflows whereas in the paper of Yang et al. (2018) the ${ }^{13} \mathrm{CO}(3-2)$ and $\mathrm{C}^{18} \mathrm{O}(3-2)$ lines 
were employed. Thus, the parameters the outflows associated with HH 137 and HH 138 are typical of high-mass stars.

\section{SUMMARY}

In this contribution, we present a multiwavelength study of the Herbig-Haro objects 137 and 138 identified by Ogura (1993) in the Carina region. High resolution Gemini $\mathrm{H}_{2}$ images allowed us to detect $2.12 \mu \mathrm{m}$ shock emissions linked to most of the optical knots composing the chain of emissions in $\mathrm{HH} 137$, as well as five new $\mathrm{H}_{2}$ knots. These new $\mathrm{H}_{2}$ emissions are designated as MHO 1629 in the on-line Catalogue of Molecular Hydrogen Emission-Line Objects (MHOs, Davis et al. 2010). We inspected Spitzer $4.5 \mu \mathrm{m}$ images finding counterparts for almost all $\mathrm{H}_{2}$ knots reported in this work. We also identified new likely shock excited regions associated with the four optical knots delineating HH 138. Moreover, a bright $4.5 \mu \mathrm{m} 0.09 \mathrm{pc}$-long arc-shaped structure, roughly located mid-way between HH 137 and HH 138 was found. This is likely associated with two Spitzer point-like sources located very close to the arc, which coincides with a peak of emission in 70 and $160 \mu \mathrm{m}$ and with the WISE J111406.96-605255.9 source. The arc-shaped structure, as well as the two point-like sources, are projected on the axis of HH 137 and $\mathrm{HH} 138$. We suggest that these stars are the exciting sources of these $\mathrm{HH}$ objects.

${ }^{12} \mathrm{CO}(3-2),{ }^{13} \mathrm{CO}(3-2)$ and $\mathrm{C}^{18} \mathrm{O}(3-2)$ molecular line data obtained with the APEX telescope allow us to identify a dense core (C1) for which we derive a LTE mass of $36 \mathrm{M}_{\odot}$. This core, also detected in the high density $\mathrm{HCO}^{+}(3-2)$ and $\mathrm{HCN}(3-2)$ molecular lines, coincides with the position of the WISE candidate exciting source.

The ${ }^{12} \mathrm{CO}(3-2)$ emission distribution over the observed region reveals molecular material associated with at least three outflows. Outflows 1 and 2 extend along the chain of optical knots forming HH 138 and HH 137 discovered by Ogura (1993) and Outflow 3 lies to the north-east. In addition, outflows 1 and 2 partially overlap on the plane of the sky. The appearance of these outflows on the plane of the sky suggests a scenario in which the driving sources, nested in the dense clump $\mathrm{C} 1$, develop two molecular outflows projected along the optical and $\mathrm{H}_{2}$ knots. Spitzer Class I sources (YSO 1 and 2) lie towards the dust clump (C1), have a projected separation of $\sim 7500 \mathrm{AU}$ and might form a wide binary system, being each star the exciting source of each outflow. Outflow 3 is projected towards a secondary weaker dust clump observed at wavelength $\geq 160 \mu \mathrm{m}$.

We estimate $\mathrm{H}_{2}$ masses $M_{\text {out }}=17,5$ and $4 \mathrm{M}_{\odot}$ for outflows 1,2 and 3 . In the cases of outflows 1 and 2, red and blue lobes are well resolved and the corresponding centers have separations of $\sim 47^{\prime \prime}$ and $81^{\prime \prime}$, which are larger than APEX HPBW of $\sim 20^{\prime \prime}$. This suggests that the inclinations angles should be small. The length of the blue lobe of outflow 2 from the terminal $\mathrm{H}_{2}$ bow shock to the proposed driving source is $0.9 \pm 0.3 \mathrm{pc}$. However, this parameter depends on the (small) inclination of the outflow with respect to the plane of the sky.

Finally, we propose a simple scenario that puts together the evidence found in this work and foresees a common origin for all shock excited emissions and the two outflows in the region, provided they are all at the same distance. This scheme also suggests the binarity of the exciting source as a natural explanation for the observed distribution of shocked emissions and/or entrained gas, which forms the blue and red-shifted lobes of the two molecular outflows in the region. Thus, this cartoon model highlights the physical connection among optical, NIR and molecular outflows in young stars.

\section{ACKNOWLEDGEMENTS}

We especially thank to Dr. Mischa Schirmer for his support and advises with the THELI's reduction package and to Dr. Rodrigo Carrasco, the instrument scientist for GSAOI, for suggestions during the observation process. We also would like to thank the anonymous referee for his/her thorough revision of the paper and his/her constructive comments and suggestions that improved the manuscript. This work was partially supported by a grant from SeCyT-UNC, Argentina. M.R and H.P.S wish to acknowledge support from CONICYT (Chile) through FONDECYT grants No1140839 and No1190684. M.R acknowledges partial support from the project BASAL PFB-06. H.P.S appreciates the financial support of a postdoctoral fellowship from SeCyT-UNC.

\section{DATA AVAILABILITY}

The data underlying this article are available in Zenodo, at https : //doi.org/10.5281/zenodo.3903751.

\section{REFERENCES}

Allen L. E., et al., 2004, ApJS, 154, 363

Arce H. G., Shepherd D., Gueth F., Lee C. F., Bachiller R., Rosen A., Beuther H., 2007, in Reipurth B., Jewitt D., Keil K., eds, Protostars and Planets V. p. 245 (arXiv: astro-ph/0603071)

Bachiller R., Tafalla M., 1999, in Lada C. J., Kylafis N. D., eds, NATO Advanced Science Institutes (ASI) Series C Vol. 540, NATO Advanced Science Institutes (ASI) Series C. p. 227

Bally J., 2016, ARA\&A, 54, 491

Barnes P. J., et al., 2011, ApJS, 196, 12

Benjamin R. A., et al., 2003, PASP, 115, 953

Beuther H., Schilke P., Sridharan T. K., Menten K. M., Walmsley C. M., Wyrowski F., 2002, A\&A, 383, 892

Bourke T. L., et al., 1997, ApJ, 476, 781

Brand J., Blitz L., 1993, A\&A, 275, 67

Carrasco E. R., et al., 2012, in Adaptive Optics Systems III. p. 84470N, doi: $10.1117 / 12.926240$

Chambers E. T., Jackson J. M., Rathborne J. M., Simon R., 2009, The Astrophysical Journal Supplement Series, 181, 360

Connelley M. S., Reipurth B., Tokunaga A. T., 2008, AJ, 135, 2496

Csengeri T., et al., 2014, A\&A, 565, A75

Cunningham N., Lumsden S. L., Moore T. J. T., Maud L. T., Mendigutía I., 2018, MNRAS, 477, 2455

Cutri R. M., et al. 2013, VizieR Online Data Catalog, 2328

Cyganowski C. J., et al., 2008, AJ, 136, 2391

Das S. R., Tej A., Vig S., Liu T., Ghosh S. K., Chandra C. H. I., 2018, A\&A, 612, A36

Davis C. J., Mundt R., Eisloeffel J., 1994, ApJ, 437, L55

Davis C. J., Kumar M. S. N., Sandell G., Froebrich D., Smith M. D., Currie M. J., 2007, MNRAS, 374, 29

Davis C. J., Gell R., Khanzadyan T., Smith M. D., Jenness T., 2010, A\&A, 511, A24

Deharveng L., et al., 2010, A\&A, 523, A6

Dewangan L. K., Ojha D. K., Zinchenko I., 2017, ApJ, 851, 140

Dobashi K., 2011, PASJ, 63, S1

Dobashi K., Uehara H., Kandori R., Sakurai T., Kaiden M., Umemoto T., Sato F., 2005, PASJ, 57, S1

Downes T. P., Cabrit S., 2007, A\&A, 471, 873

Du F., Yang J., 2008, ApJ, 686, 384 

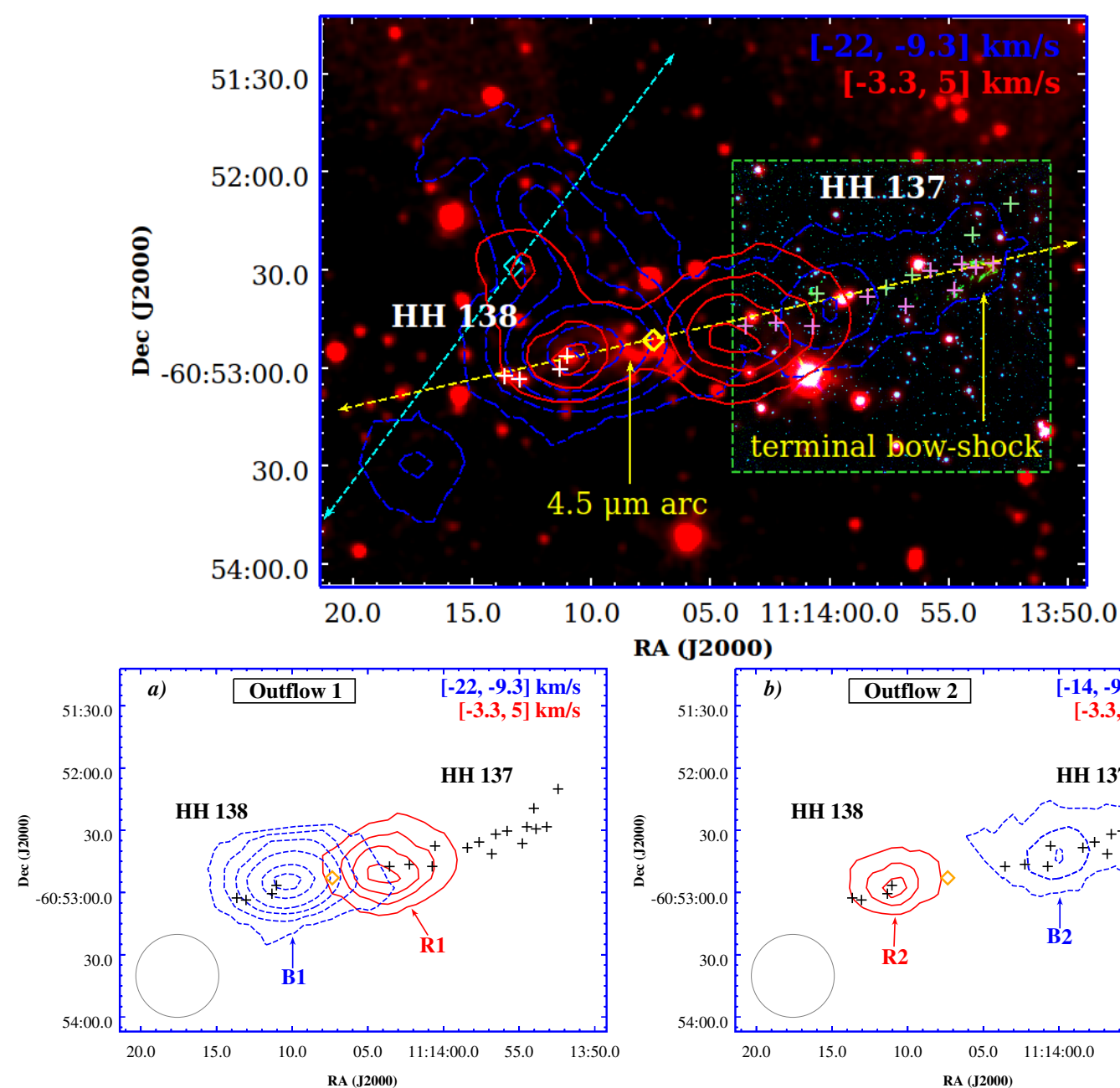

RA (J2000)
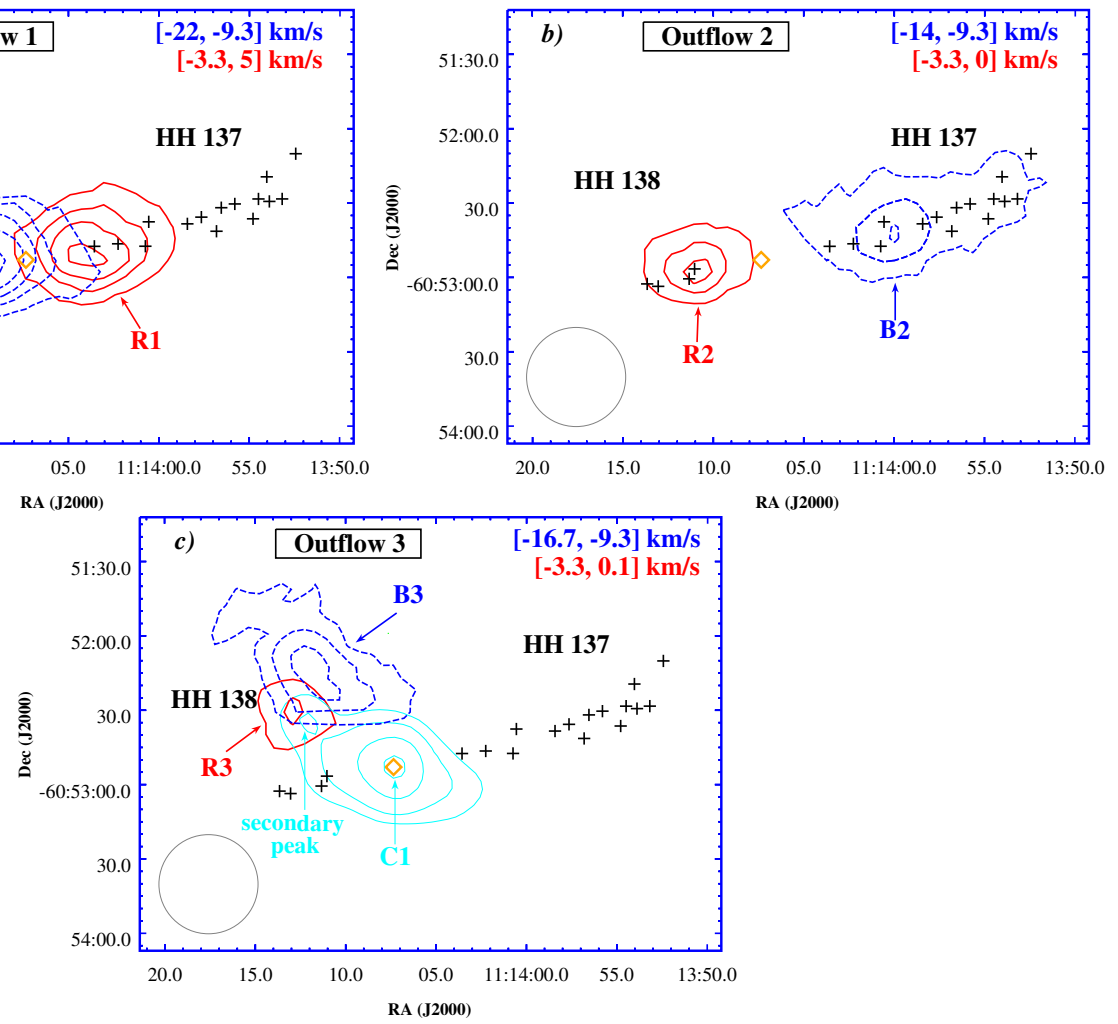

Figure 11. Upper panel: Blueshifted and redshifted wings of the ${ }^{12} \mathrm{CO}$ molecular line emission in the velocity ranges of $[-22,-9.3] \mathrm{km} \mathrm{s}^{-1}$ and $[-3.3,5] \mathrm{km} \mathrm{s}^{-1}$, respectively, superimposed on a combined three band images: $\mathrm{K}$ (blue), $\mathrm{H}_{2}$ (green), and $4.5 \mu \mathrm{m}$ (red). The dashed blue lines correspond to 2, $612,20,34$ and $44 \mathrm{~K} \mathrm{~km} \mathrm{~s}^{-1}$ and the continuous red line to 4, 8, 11, and $14 \mathrm{~K} \mathrm{~km} \mathrm{~s}^{-1}$. The magenta and light green crosses indicate the positions of optical knots of HH 137 (Ogura 1993) and new $\mathrm{H}_{2}$ knots from Section 3. The white crosses mark the optical knots of HH 138 from Ogura (1993). The dashed green line box underlines the field observed with Gemini. The terminal $\mathrm{H}_{2}$ bow shock as well as the $4.5 \mu \mathrm{m}$ arc-like structure are indicated with yellow arrows. The double arrow dashed yellow line marks the cutting line used to make the Position-Velocity diagram in Figure 12, upper panel, indicating the outflow direction from coordinates RA.,Dec. (J2000) $=(11: 13: 49.605,-60: 52: 21.69)$ to $(11: 14: 20.693,-60: 53: 13.05)$. The yellow diamond marks the location of the Spitzer and WISE exciting sources discussed in Section 5, chosen as the center of offset positions shown in Figure 12 (upper panel). The double arrow dashed cyan line indicates the cutting line used to make the Position-Velocity diagram in Figure 12 (lower panel), showing the outflow direction from coordinates RA.,Dec. $(J 2000)=(11: 14: 21.222,-60: 53: 46.48)$ to $(11: 14: 06.512,-60: 51: 23.76)$. The cyan diamond marks the position of the offset center in Figure 12, lower panel, located at RA.,Dec. (J2000) $=(11: 14: 12.054,-60: 52: 35.50)$. The center of this offset position lies 10.8 " north-west of the secondary dust peak in Figure 5. Middle and bottom panels: Blueshifted and redshifted lobes associated to the outflow 1 (middle left panel), outflow 2 (middle right panel) and outflow 3 (bottom panel) in the velocity ranges indicated in the upper right corner of each panel. The orange diamond marks the location of the suggested exciting stars (see Section 5). In the bottom panel c), the cyan contours are the $160 \mu \mathrm{m}$ dust emissions associated with clump C1 and a secondary dust peak (see Figure 5). The HPBW is shown in the lower left corner of each panel. 


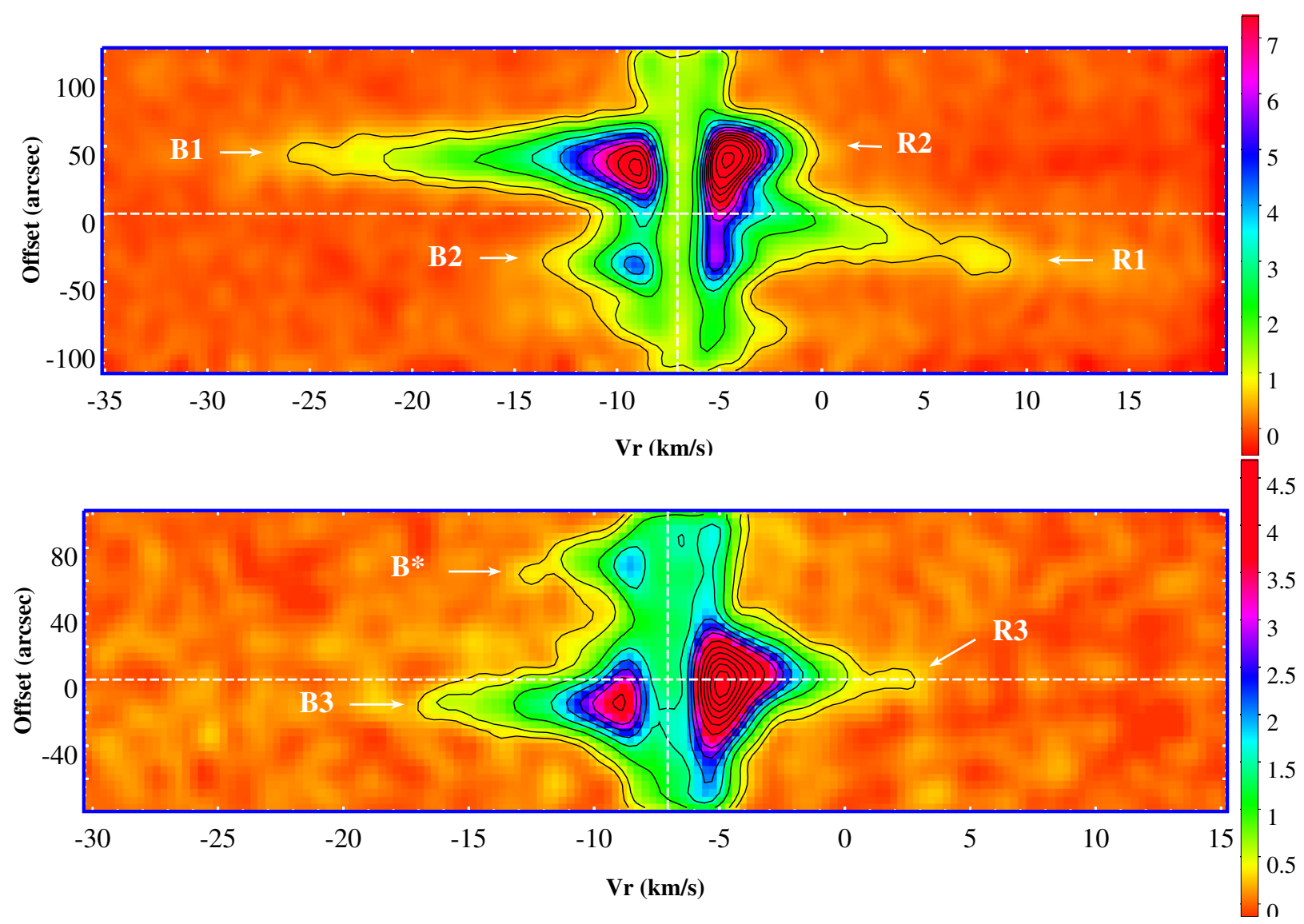

Figure 12. Position-Velocity (PV) diagram of the ${ }^{12} \mathrm{CO}(3-2)$ emission along two transverse cuts shown in Figure 11. Upper panel: Cut along the double arrow dashed yellow line in Figure 11. Offset coordinates are referred to the suggested exiting sources positions (see Section 5), located at RA.,Dec. $(J 2000)=(11: 14: 07.397,-60: 52: 51.39)$. The black contours correspond to 0.6, 1, 2, 3, 4, 5, 6, 7, 8, 9, 10, 12 and 14 K. Lower panel: Cut along the double arrow dashed cyan line in Figure 11. Offset coordinates are referred to a position (RA.,Dec. (J2000) $=(11: 14: 13.240,-60: 52: 35.29 .03))$ on the cutting line, indicated with a cyan diamond, 10.8 " to the north-west of the position of the secondary peak. The black contours correspond to $0.3,0.5,1,1.5,2,2.5$, $3,4,5,6,7,8$ and $9 \mathrm{~K}$. The blueshifted emission identified as $\mathrm{B}^{*}$, corresponds to the blue contours at the south-east corner in Figure 11 . The color scale is indicated at the right. The horizontal dashed white line marks the zero offset position. The vertical dashed white line corresponds to the velocity of the central depression detected in the ${ }^{12} \mathrm{CO}$ molecular line (see Figure 6).

Table 4. Parameters of the outflows.

\begin{tabular}{lcccccc}
\hline & \multicolumn{2}{c}{ Outflow 1 } & \multicolumn{2}{c}{ Outflow 2 } & \multicolumn{2}{c}{ Outflow 3 } \\
Parameters & \multicolumn{1}{c}{ B1 } & R1 & B2 & R2 & B3 & R3 \\
\hline Velocity range $\left[\mathrm{km} \mathrm{s}^{-1}\right]$ & {$[-22,-9.3]$} & {$[-3.3,5]$} & {$[-14,-9.3]$} & {$[-3.3,0]$} & {$[-16.7,-9.3]$} & {$[-3.3,0.1]$} \\
$\int I_{12} C O d v\left[\mathrm{~km} \mathrm{~s}^{-1}\right]$ & $2272 \pm 48$ & $924 \pm 30$ & $654 \pm 26$ & $421 \pm 21$ & $658 \pm 26$ & $174 \pm 13$ \\
$\mathrm{~V}_{\text {max }}\left[\mathrm{km} \mathrm{s}^{-1}\right]$ & $15.7 \pm 0.3$ & $11.3 \pm 0.3$ & $7.7 \pm 0.3$ & $5.8 \pm 0.3$ & $10.4 \pm 0.3$ & $6.4 \pm 0.3$ \\
$\mathrm{r}[\mathrm{pc}]$ & $0.4 \pm 0.2$ & $0.4 \pm 0.2$ & $0.9 \pm 0.3$ & $0.3 \pm 0.2$ & $0.7 \pm 0.2$ & $0.4 \pm 0.2$ \\
$M_{\text {out }}\left[\mathrm{M}_{\odot}\right]$ & $12 \pm 8$ & $5 \pm 3$ & $3 \pm 2$ & $2 \pm 1$ & $3 \pm 2$ & $0.9 \pm 0.6$ \\
$p\left[\mathrm{M}_{\odot} \mathrm{km} \mathrm{s}^{-1}\right]$ & $182 \pm 122$ & $53 \pm 36$ & $26 \pm 17$ & $12 \pm 8$ & $35 \pm 23$ & $6 \pm 4$ \\
$E\left[10^{45} \mathrm{erg}\right]$ & $28 \pm 19$ & $6 \pm 4$ & $2 \pm 1$ & $0.7 \pm 0.5$ & $4 \pm 2$ & $0.4 \pm 0.2$ \\
$t\left[10^{4} \mathrm{yr}\right]$ & $3 \pm 1$ & $4 \pm 2$ & $12 \pm 4$ & $6 \pm 3$ & $7 \pm 2$ & $7 \pm 3$ \\
$\dot{M}_{\text {out }}\left[10^{-5}{\left.\mathrm{M} \odot \mathrm{yr}^{-1}\right]}_{F_{m}\left[10^{-4} \mathrm{M}_{\odot} \mathrm{km} \mathrm{s}^{-1} \mathrm{yr}^{-1}\right]}^{41 \pm 34}\right.$ & $12 \pm 10$ & $3 \pm 2$ & $3 \pm 3$ & $5 \pm 4$ & $1 \pm 1$ \\
$L_{m}\left[\mathrm{~L}_{\odot}\right]$ & $85 \pm 53$ & $13 \pm 11$ & $2 \pm 2$ & $2 \pm 2$ & $5 \pm 4$ & $0.8 \pm 0.6$ \\
\hline
\end{tabular}

Dutra C. M., Bica E., 2002, A\&A, 383, 631

Elia D., et al., 2013, ApJ, 772, 45

Elia D., et al., 2017, MNRAS, 471, 100

Erben T., et al., 2005, Astronomische Nachrichten, 326, 432

Evans Neal J. I., 1999, ARA\&A, 37, 311

Fedriani R., et al., 2018, A\&A, 616, A126
Feitzinger J. V., Stuewe J. A., 1984, Astronomy and Astrophysics Supplement Series, 58, 365

Ferrero L. V., Gómez M., Gunthard G., 2015, Boletin de la Asociacion Argentina de Astronomia, La Plata, Argentina, 57, 126

Graham M. F., Meaburn J., Redman M. P., 2003, MNRAS, 343, 419

Griffin M. J., et al., 2010, A\&A, 518, L3 


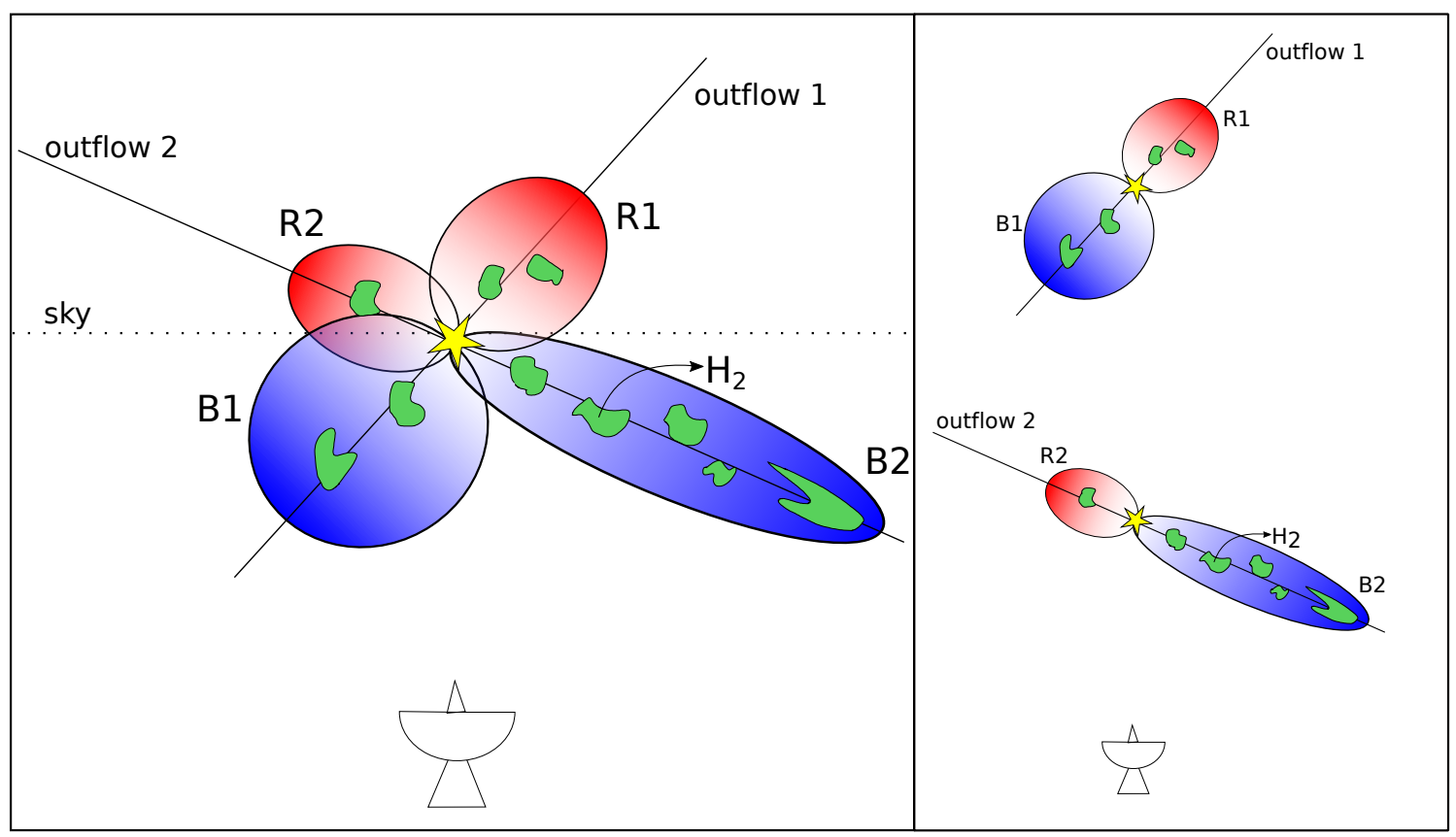

Figure 13. Schematic representation of outflows 1 and 2 with respect to the plane of the sky. In the left panel, the outflows are at the same distance and the driving sources form a binary star. In the right panel, $\mathrm{x}$ the outflows are at different distances and no physical association between the exciting stars exists.

Güsten R., Nyman L. Å., Schilke P., Menten K., Cesarsky C., Booth R., 2006, A\&A, 454, L13

Hartley M., Tritton S. B., Manchester R. N., Smith R. M., Goss W. M., 1986, A\&AS, 63, 27

Hatchell J., Fuller G. A., Richer J. S., 2007, A\&A, 472, 187

Hildebrand R. H., 1983, QJRAS, 24, 267

Jackson J. M., Finn S. C., Rathborne J. M., Chambers E. T., Simon R., 2008, ApJ, 680, 349

Kavars D. W., Dickey J. M., McClure-Griffiths N. M., Gaensler B. M., Green A. J., 2005, ApJ, 626, 887

Koenig X. P., Leisawitz D. T., Benford D. J., Rebull L. M., Padgett D. L., Assef R. J., 2012, ApJ, 744, 130

König C., et al., 2017, A\&A, 599, A139

Lada C. J., 1985, ARA\&A, 23, 267

Lee K. I., et al., 2016, ApJ, 820, L2

Liu H.-L., et al., 2017, A\&A, 602, A95

Marsh K. A., Whitworth A. P., Lomax O., 2015, MNRAS, 454, 4282

Marsh K. A., et al., 2017, MNRAS, 471, 2730

Maud L. T., Moore T. J. T., Lumsden S. L., Mottram J. C., Urquhart J. S., Hoare M. G., 2015, MNRAS, 453, 645

McGregor P., et al., 2004, in Moorwood A. F. M., Iye M., eds, Proc. SPIEVol. 5492, Ground-based Instrumentation for Astronomy. pp 1033-1044, doi:10.1117/12.550288

McGroarty F., Ray T. P., Froebrich D., 2007, A\&A, 467, 1197

McLeod A. F., Reiter M., Kuiper R., Klaassen P. D., Evans C. J., 2018, Nature, 554, 334

Moffat A. F. J., Vogt N., 1975, Astronomy and Astrophysics Supplement Series, 20, 155

Molinari S., et al., 2010, PASP, 122, 314

Neichel B., et al., 2014a, MNRAS, 440, 1002

Neichel B., Lu J. R., Rigaut F., Ammons S. M., Carrasco E. R., Lassalle E., 2014b, MNRAS, 445, 500

Noriega-Crespo A., Garnavich P. M., 2001, AJ, 122, 3317

Ogura K., 1993, MNRAS, 262, 735

Ossenkopf V., Henning T., 1994, A\&A, 291, 943

Parker Q. A., et al., 2005, MNRAS, 362, 689

Planck Collaboration et al., 2016, A\&A, 594, A28

Poglitsch A., et al., 2010, A\&A, 518, L2

Pomohaci R., Oudmaijer R. D., Goodwin S. P., 2019, MNRAS, 484, 226
Povich M. S., et al., 2013, ApJS, 209, 31

Reach W. T., et al., 2006, AJ, 131, 1479

Reipurth B., Bally J., 2001, ARA\&A, 39, 403

Reipurth B., Heathcote S., Yu K. C., Bally J., Rodríguez L. F., 2000, ApJ, 534,317

Riaz B., Briceño C., Whelan E. T., Heathcote S., 2017, ApJ, 844, 47

Rigaut F., et al., 2014, MNRAS, 437, 2361

Saldaño H. P., Rubio M., Cappa C. E., Gómez M., 2019, MNRAS, 487, 2881

Sandqvist A., 1977, A\&A, 57, 467

Sanhueza P., Garay G., Bronfman L., Mardones D., May J., Saito M., 2010, ApJ, 715, 18

Schirmer M., 2013, ApJS, 209, 21

Schirmer M., Carrasco E. R., Pessev P., Garrel V., Winge C., Neichel B., Vidal F., 2015, ApJS, 217, 33

Schuller F., et al., 2009, A\&A, 504, 415

Shirley Y. L., 2015, PASP, 127, 299

Simon R., Jackson J. M., Rathborne J. M., Chambers E. T., 2006a, ApJ, 639, 227

Simon R., Rathborne J. M., Shah R. Y., Jackson J. M., Chambers E. T., 2006b, ApJ, 653, 1325

Smith M. D., Rosen A., 2005, MNRAS, 357, 1370

Smith H. A., Hora J. L., Marengo M., Pipher J. L., 2006, ApJ, 645, 1264

Steppe H., 1977, Astronomy and Astrophysics Supplement Series, 27, 415

Targon C. G., Rodrigues C. V., Cerqueira A. H., Hickel G. R., 2011, ApJ, 743,54

Tóth L. V., et al., 2014, PASJ, 66, 17

Tsinganos K., Ray T., Stute M., 2009, Astrophysics and Space Science Proceedings, 13

Vassilev V., et al., 2008, A\&A, 490, 1157

Walsh A. J., Purcell C. R., Longmore S. N., Breen S. L., Green J. A., Harvey-Smith L., Jordan C. H., Macpherson C., 2014, MNRAS, 442, 2240

Watson C., et al., 2008, ApJ, 681, 1341

Watson C., Hanspal U., Mengistu A., 2010, ApJ, 716, 1478

Whelan E. T., Riaz B., Rouzé B., 2018, A\&A, 610, L19

Wright E. L., et al., 2010, AJ, 140, 1868

Wu P.-F., Takakuwa S., Lim J., 2009, ApJ, 698, 184 
Yamaguchi R., Saito H., Mizuno N., Mine Y., Mizuno A., Ogawa H., Fukui Y., 1999, PASJ, 51, 791

Yang A. Y., Thompson M. A., Urquhart J. S., Tian W. W., 2018, ApJS, 235,

Zhou S., Evans II N. J., 1994, in Clemens D. P., Barvainis R., eds, Astronomical Society of the Pacific Conference Series Vol. 65, Clouds, Cores, and Low Mass Stars. p. 183

de Villiers H. M., et al., 2014, MNRAS, 444, 566

This paper has been typeset from a $\mathrm{T}_{\mathrm{E}} \mathrm{X} / \mathrm{L} \mathrm{T} \mathrm{E} \mathrm{X}$ file prepared by the author. 